\title{
ON THE DEPENDENCE OF ANALYTIC SOLUTIONS OF PARTIAL DIFFERENTIAL EQUATIONS ON THE RIGHT-HAND SIDE
}

\author{
SIEGFRIED MOMM
}

\begin{abstract}
Given a nonzero polynomial $P(z)=\sum_{|\alpha| \leq m} a_{\alpha} z^{\alpha}$ on $C^{N}$, Martineau proved in the 1960 s that for each convex domain $G$ of $C^{N}$ the partial differential operator $P(D) f=\sum_{|\alpha| \leq m} a_{\alpha} f^{(\alpha)}$ acting on the Fréchet space $A(G)$ of all analytic functions on $G$ is surjective. In the present paper it is investigated whether solutions $f$ of the equation $P(D) f=g$ can be chosen as $f=R(g)$ with a continuous linear operator $R: A(G) \rightarrow A(G)$. For bounded $G$ we give a necessary and sufficient condition for the existence of such an $R$.
\end{abstract}

\section{INTRODUCTION}

Given a nonzero polynomial $P(z)=\sum_{|\alpha| \leq m} a_{\alpha} z^{\alpha}$ on $\mathbb{C}^{N}$, Martineau [19] proved that for each convex domain $G$ of $\mathbb{C}^{N}$ and for each function $g$ analytic on $G$, there is a solution $f$ analytic on $G$ of the partial differential equation

$$
P(D) f:=\sum_{|\alpha| \leq m} a_{\alpha} f^{(\alpha)}=g \text {. }
$$

If the space $A(G)$ of all analytic functions on $G$ is endowed with the natural Fréchet space topology, this result says that $P(D): A(G) \rightarrow A(G)$ is a surjective continuous linear map. For $G=\mathbb{C}^{N}$, for which the latter result is due to Malgrange [18], it is an old theorem of Kiselman [14] and Trèves [33] that for a fixed noncharacteristic hyperplane $L$ in $\mathbb{C}^{N}$, the unique solution $f=R(g)$ of the analytic Cauchy problem with zero initial data on $L$ defines a continuous linear map $R: A(G) \rightarrow A(G)$ with

$$
P(D) \circ R=\mathrm{id}_{A(G)} .
$$

$R$ is called a solution operator for $P(D)$ on $A(G)$. Thus $\mathbb{C}^{N}$ is one particular example of a convex domain $G$, such that each nonzero partial differential operator (even of infinite order, as it has been proved by Meise and Taylor [20]) admits a solution operator on $A(G)$. These domains have been characterized in [28]. If $G$ is in addition bounded and contains the origin and if $H$ denotes the support function of the convex set $G$, it has been proved in [28] that the domain $G$ belongs to the class in question if and only if $v_{H}=H$ on a neighborhood of the origin. Here $v_{H}: \mathbb{C}^{N} \rightarrow \mathbb{R}_{+}$is the largest plurisubharmonic function on $\mathbb{C}^{N}$ with $v_{H} \leq H$ and such that $v_{H}-\log (1+|z|)$ is bounded from above. The particular property fails for instance if $G$ is a polyhedron.

Received by the editors March 10, 1993 and, in revised form, November 29, 1993.

1991 Mathematics Subject Classification. Primary 32F05, 35C99; Secondary 35E99, 35R50. 
In the present paper for a given bounded convex domain $G$ of $\mathbb{C}^{N}$ which contains the origin and with support function $H$, we give a criterion for $P(D)$ to admit a solution operator on $A(G)$. If $P$ is a homogeneous polynomial our criterion can be phrased as an appropriate lower bound for the extremal plurisubharmonic function $v_{H}$ on the zero variety $V(P):=\left\{z \in \mathbb{C}^{N} \mid P(\bar{z})=0\right\}$ :

Theorem. Let $P$ be a nonzero homogeneous polynomial on $\mathbb{C}^{N}$. The partial differential operator $P(D)$ admits a solution operator on $A(G)$ if and only if there is $\delta>0$ such that $v_{H} \geq v_{H, V(P), \delta}$ on $V(P)$ in a neighborhood of the origin. Here $v_{H, V(P), \delta}$ is the largest plurisubharmonic function on $V(P)$ with $v_{H, V(P), \delta} \leq H$ and such that $v_{H, V(P), \delta}-\delta \log (1+|z|)$ is bounded from above.

We introduce the cone $\Gamma_{H}:=\left\{t a \mid t \geq 0, a \in \mathbb{C}^{N}, v_{H}(a)=H(a)\right\} \subset \mathbb{C}^{N}$ and show that in the case of a polyhedron $G$, this criterion roughly says that $v_{H} \mid V(P)$ is a "maximal plurisubharmonic function" on $V(P) \backslash \Gamma_{H}$ (for the definition, see Proposition 3.10). From our theorem we deduce in $\S 3$ the following result which looks like an analogue of the classical one of Grothendieck for elliptic partial differential operators on $C^{\infty}(\Omega)$ for open sets $\Omega$ in $\mathbf{R}^{N}$ :

Corollary. If $P$ is a nonconstant polynomial on $\mathbb{C}^{N}$ with principal part $P_{m}$ such that $V\left(P_{m}\right) \cap \Gamma_{H}=\{0\}$, then the partial differential operator $P(D)$ admits no solution operator on $A(G)$.

In the most simple nontrivial case, a characterization of partial differential operators with a solution operator is given by

Proposition. Let $G=G_{1} \times G_{2} \subset \mathbb{C}^{2}$ be a polyhedron. Let $P$ be a nonconstant polynomial on $\mathbb{C}^{2}$ with principal part $P_{m}$. Let the numbers $\alpha_{1}, \ldots, \alpha_{n} \in \mathbb{C} \backslash\{0\}$ be determined by the representation

$$
P_{m}(z)=c z_{1}^{l_{1}} z_{2}^{l_{2}} \prod_{j=1}^{n}\left(z_{1}-\alpha_{j} z_{2}\right), \quad z \in \mathbb{C}^{2},
$$

for appropriate $c \in \mathbb{C} \backslash\{0\}$ and $l_{1}, l_{2} \in \mathbb{N}_{0}$. Then $P(D)$ admits a solution operator on $A(G)$ if and only if

$$
\alpha_{j} \Gamma_{1}=\Gamma_{2}, \quad j=1, \ldots, n .
$$

Here $\Gamma_{i} \subset \mathbb{C}$ is the cone generated by the outer normals to the faces of $G_{i}$, $i=1,2$.

In a certain sense we continue investigations of Kiselman [14], who gave sufficient conditions for the solvability of the analytic Cauchy problem in $A(G)$ with zero initial data on a noncharacteristic hyperplane passing through the origin. Our general criterion for $P(D)$ to admit a solution operator on $A(G)$ can be interpreted as a type of Phragmén-Lindelöf principle for analytic (or plurisubharmonic) functions on the zero variety $V(P)$. A condition like this was introduced by Hörmander [11] to characterize the surjective partial differential operators on the space of real analytic functions on a given convex domain of $\mathbf{R}^{N}$. Meise, Taylor, and Vogt [21] used a similar Phragmén-Lindelöf condition to characterize the partial differential operators which admit a solution operator on $C^{\infty}(\Omega)$ for a given convex domain $\Omega$ of $R^{N}$. In contrast to the situation which is considered in the present paper, starting with Grothendieck 
many authors investigated whether $P(D)$ admits a solution operator on $C^{\infty}(\Omega)$ for a given domain $\Omega$ of $\mathbf{R}^{N}$. For references we refer to the recent systematic treatise of this problem in [21].

In this paper we investigate the existence of a solution operator on $A(G)$ not only for partial differential operators but also for the larger class of socalled differential operators of infinite order. Our paper is divided into three sections. Section 1: The precise knowledge of the locally convex structure of the space $A(G)$ which is due to Zaharjuta [37], the procedure of Vogt [34] (and Khenkin and Mityagin, see [34]) of constructing a basis in each nuclear Fréchet space which admits the linear topological invariants $(\bar{\Omega})$ and $(\underline{D N})$, and the observation of Langenbruch [17] that certain versions $(\bar{\Omega})_{t}$ and $(\underline{D N})_{t}$ of these invariants give a nice estimation of this basis; all these ingredients allow one to apply Vogt's splitting theorem [35] for short exact sequences of power series spaces of finite type. This shows that a differential operator $P(D)$ admits a solution operator if and only if $\operatorname{ker} P(D)$ satisfies the property $(\bar{\Omega})_{t}$ for a certain natural system of norms on $\operatorname{ker} P(D)$. In the remaining part of this paper we evaluate this criterion. Section 2 deals with differential operators of infinite order. Section 3 is devoted to the investigation of partial differential operators.

\section{A CRITERION FOR A DIFFERENTIAL OPERATOR TO ADMIT A SOLUTION OPERATOR}

In this section we derive a general criterion for the existence of a solution operator for a given differential operator of infinite order. This criterion will be evaluated in the sections which will follow.

Throughout this paper, for all $z, w \in \mathbb{C}^{N}$ and $r>0$, we will use the following abbreviations: $\langle w, z\rangle:=\sum_{j=1}^{N} w_{j} \bar{z}_{j},|z|:=\langle z, z\rangle^{1 / 2}, U(z, r):=$ $\left\{w \in \mathbb{C}^{N}|| w-z \mid<r\right\}, U(r):=U(0, r), B(z, r):=\left\{w \in \mathbb{C}^{N}|| w-z \mid \leq r\right\}$, $B(r):=B(0, r)$, and $S:=\partial B(1), \mathbf{R}_{+}:=[0, \infty[$. For standard results from functional analysis, we refer to the book of Meise and Vogt [23].

1.1. Notation. For the sequel, we fix a bounded convex domain $G$ of $\mathbb{C}^{N}$ which contains the origin. Let $H$ be its support function, i.e.,

$$
H(z)=\sup _{w \in G} \operatorname{Re}\langle w, z\rangle, \quad z \in \mathbb{C}^{N} .
$$

Let $g: G \rightarrow[-\infty, 0[$ denote the pluricomplex Green function of $G$ with pole at the origin, i.e.,

$$
g(z):=\sup _{u} u(z), \quad z \in G,
$$

where the supremum is taken over all plurisubharmonic functions $u: G \rightarrow$ $[-\infty, 0[$ with $u(w) \leq \log |w|+O(1)$ as $w \rightarrow 0$ (see Klimek [16]). For $x<0$ we consider the sets $G_{x}:=\{z \in G \mid g(z)<x\}$. Due to a result of Lempert these sets are convex (see [27, Lemma 1.2]). Let $H_{x}$ denote its support function.

We will frequently apply the following properties of $\left(H_{x}\right)_{x<0}$ : For all $x<$ $y<0$ there are $\delta, \varepsilon>0$ such that

$$
\delta|z| \leq H_{x}(z) \text { and } H_{x}(z)+\varepsilon|z| \leq H_{y}(z), \quad z \in \mathbb{C}^{N} .
$$

This holds because the origin is contained in the interior of $G_{x}$ and since $G_{x}$ is relatively compact in $G_{y}$. 
1.2. Function spaces. For each domain $D$ of $\mathbb{C}^{N}$, let $A(D)$ denote the set of all analytic functions on $D$. We endow the Fréchet space $A(G)$ of all analytic functions on $G$ with the norms

$$
|f|_{x}:=\sup _{z \in G_{x}}|f(z)|, \quad f \in A(G), x<0 .
$$

By $A_{H}$ we denote the (LB)-space

$$
\begin{gathered}
A_{H}=\left\{f \in A\left(\mathbb{C}^{N}\right) \mid\|f\|_{x}<\infty \text { for some } x<0\right\}, \\
\|f\|_{x}:=\sup _{z \in \mathbb{C}^{N}}|f(z)| \exp \left(-H_{x}(\bar{z})\right) .
\end{gathered}
$$

Thus $A_{H}$ consists of all entire functions $f$ on $\mathbb{C}^{N}$ satisfying the estimate

$$
|f(z)| \leq C \exp (\eta H(\bar{z})), \quad z \in \mathbb{C}^{N},
$$

for some $C>0$ and some $0<\eta<1$.

By $A^{0}$ we denote the Fréchet space of all entire functions $P$ on $C^{N}$ such that for all $x<0$

$$
|P|_{x}:=\sup _{z \in \mathrm{C}^{N}}|P(z)| \exp (x|z|)<\infty .
$$

1.3. Differential operators. We fix some $P \in A^{0} \backslash\{0\}, P(z)=\sum_{\alpha \in \mathbb{N}_{0}^{N}} a_{\alpha} z^{\alpha}$ for $z \in \mathbb{C}^{N}$. By Martineau [19, Theorem 7], the following continuous linear map $P(D): A(G) \rightarrow A(G)$ is surjective:

$$
P(D) f:=\sum_{\alpha \in \mathbb{N}_{0}^{N}} a_{\alpha} f^{(\alpha)}, \quad f \in A(G) .
$$

Our aim is to decide whether there is a solution operator for $P(D)$ on $A(G)$, i.e., a continuous linear map $R: A(G) \rightarrow A(G)$ with $P(D) \circ R=\mathrm{id}_{A(G)}$. To this end we want to apply a result of Vogt [35]. This requires some preparation.

1.4. Notation. For a Fréchet space $E$ with a system of seminorms $\left(|\cdot|_{x}\right)_{x<0}$, we consider the dual "norms"

$$
|\mu|_{x}^{*}:=\sup \left\{\left.|\mu(f)||f \in E,| f\right|_{x} \leq 1\right\} \in[0, \infty], \quad \mu \in E^{\prime}, x<0 .
$$

For $P \in A^{0}$, let $q: A_{H} \rightarrow A_{H} /\left(P \cdot A_{H}\right)$ denote the quotient map. For $x<0$ and $f \in A_{H}$ with $\|f\|_{x}<\infty$, we define

$$
\|q(f)\|_{x}:=\inf \left\{\|g\|_{x} \mid g \in A_{H},\|g\|_{x}<\infty, q(g)=q(f)\right\} .
$$

1.5. Laplace transform and duality. The Laplace transform $\mathscr{F}: A(G)^{\prime} \rightarrow A_{H}$, defined by $\mathscr{F}(\mu)(z):=\mu\left(e^{(\cdot, \bar{z})}\right), \mu \in A(G)^{\prime}, z \in \mathbb{C}^{N}$, is an isomorphism of (LB)-spaces. Moreover, for all $x_{1}<x_{2}<0$ there is $C>0$ with

(i) $\|\mathscr{F}(\mu)\|_{x_{2}} \leq C|\mu|_{x_{1}}^{*}$ for all $\mu \in A(G)^{\prime}$ and

(ii) $\left|\mathscr{F}^{-1}(f)\right|_{x_{2}}^{*} \leq C\|f\|_{x_{1}}$ for all $f \in A_{H}$.

Let $P \in A^{0} \backslash\{0\}$. Identifying $A(G)^{\prime}$ and $A_{H}$ by $\mathscr{F}$, the transposed map $P(D)^{\mathrm{t}}$ : $A(G)^{\prime} \rightarrow A(G)^{\prime}$ is the operator $M_{P}: A_{H} \rightarrow A_{H}, M_{P}(f)=P \cdot f$. Via duality theory for (FS)-spaces, $P(D)$ is onto if and only if $P \cdot A_{H}$ is a closed subspace of $A_{H}$, the latter being true by Martineau's result. Furthermore, the canonical map

$$
A_{H} /\left(P \cdot A_{H}\right) \rightarrow(\operatorname{ker} P(D))^{\prime}, \quad q(f) \mapsto \mathscr{F}^{-1}(f) \mid \operatorname{ker} P(D),
$$


is an isomorphism of (LB)-spaces. More precisely, for all $x_{1}<x_{2}<0$ there is $C>0$ such that for each $f \in A_{H}$

(i) $\|q(f)\|_{x_{2}} \leq C\left|F^{-1}(f)\right|_{x_{1}}^{*}$ if $\left|F^{-1}(f)\right|_{x_{1}}^{*}<\infty$ and

(ii) $\left|\mathscr{F}^{-1}(f)\right|_{x_{2}}^{*} \leq C\|q(f)\|_{x_{1}}$ if $\|f\|_{x_{1}}<\infty$.

Proof. These are well-known properties of the Laplace transform (see, e.g., Hörmander [10, Theorem 4.5.3]) and of duality theory (see also [24, Proposition 2.10]).

\subsection{Division theorem.}

(a) For each $\varepsilon>0$ there is $x<0$ such that for all $\delta>0$ and $K>0$ there is $R>0$ such that for all $P \in A^{0}$ with $|P(0)| \geq \delta$ and $|P|_{x} \leq K$ the following holds: For each $z \in \mathbb{C}^{N}$ with $|z| \geq R$ there is $w \in \mathbb{C}^{N}$ with $|w-z| \leq \varepsilon|z|$ and $|P(w)| \geq \exp (-\varepsilon|w|)$.

(b) For all $x_{1}<x_{2}<0$ there is $x<0$ such that for all $\delta>0$ and $K>0$ there is $C>0$ such that for all $P \in A^{0}$ with $|P(0)| \geq \delta$ and $|P|_{x} \leq K$ the following holds. For all $f \in A_{H}$ with $g:=f / P \in A\left(\mathbb{C}^{N}\right)$ and $\|f\|_{x_{1}}<\infty$ we get $\|g\|_{x_{2}} \leq C\|f\|_{x_{1}}$.

Proof. (a) follows from Martineau [19, Lemme 15]. (b) follows from (a), by Hörmander [9, Lemma 3.2].

We give a quantitative version of Martineau's result:

1.7. Proposition. $P(D): A(G) \rightarrow A(G)$ is a continuous surjective linear map. More precisely, for all $x_{1}<x_{2}<0$ there is $C>0$ with

(i) $|P(D) f|_{x_{1}} \leq C|f|_{x_{2}}$ for all $f \in A(G)$ and

(ii) $\inf \left\{|h|_{x_{1}} \mid h \in A(G), P(D) h=g\right\} \leq C|g|_{x_{2}}$ for all $g \in A(G)$.

Proof. The straightforward estimate (i) can be found in [26, Proposition 1.5]. To check (ii), we may proceed as in [24, Proposition 2.5], where we control the estimates while applying duality theory. The several variables substitute for the estimates for the Laplace transform [24, Proposition 1.7] is 1.5. The several variables substitute for the division result [24, Corollary 2.4$]$ is $1.6(\mathrm{~b})$.

1.8. Definition. Let $\beta=\left(\beta_{j}\right)_{j \in \mathrm{N}}$ be a nondecreasing unbounded sequence of nonnegative numbers. The Fréchet space

$$
\begin{gathered}
\Lambda_{0}(\beta):=\left\{y=\left.\left(y_{j}\right)_{j \in \mathbb{N}} \in \mathbb{C}^{N}|| y\right|_{x}<\infty \text { for all } x<0\right\}, \\
|y|_{x}:=\sum_{j \in \mathbb{N}}\left|y_{j}\right| e^{x \beta_{j}},
\end{gathered}
$$

is called a power series space of finite type. To simplify the notation, each finitedimensional space will also be called by this name.

1.9. Theorem (Zaharjuta [37, Theorem 4.5]). There is a nondecreasing unbounded sequence $\beta$ of nonnegative numbers and an isomorphism $B: A(G) \rightarrow$ $\Lambda_{0}(\beta)$ such that for all $x_{1}<x_{2}<0$ there is $C>0$ with

(i) $|B(g)|_{x_{1}} \leq C|g|_{x_{2}}$ for all $g \in A(G)$ and

(ii) $\left|B^{-1}(y)\right|_{x_{1}} \leq C|y|_{x_{2}}$ for all $y \in \Lambda_{0}(\beta)$.

We recall some definitions from Vogt [35]. 
1.10. Definition. Let $E$ and $F$ be Fréchet spaces with fixed fundamental systems of seminorms which will both be denoted by $\left(|\cdot|_{x}\right)_{x<0}$. Let $A: E \rightarrow F$ be a continuous linear map. We consider its characteristic of continuity

$$
\sigma_{A}(x):=\inf \left\{y<\left.0\left|\sup _{|e|_{y} \leq 1}\right| A e\right|_{x}<\infty\right\}, \quad x<0 .
$$

$A$ is called linearly tame (tame) if there are $C \geq 1 \quad(C=1)$ and $x_{0}<0$ with $\sigma_{A}(x) \leq C x$ for all $x_{0} \leq x<0$.

A short exact sequence $0 \rightarrow F \stackrel{l}{\rightarrow} G \stackrel{q}{\rightarrow} E \rightarrow 0$ of Fréchet spaces with linear continuous maps $l$ and $q$, each space equipped with a fixed fundamental system of seminorms, is called linear-tame exact (tame exact) if the canonical maps $j: F \rightarrow \operatorname{ker} q, \bar{q}: G / \operatorname{ker} q \rightarrow E$, and their inverses are linearly tame (tame) maps $(\operatorname{ker} q$ and $G / \operatorname{ker} q$ are equipped with the subspace and quotient seminorms, respectively).

$E$ and $F$ will be called linear-tame (tame) isomorphic if there is an isomorphism $A: E \rightarrow F$ such that $A$ and $A^{-1}$ are linearly tame (tame).

1.11. Remark. By Proposition 1.7, the short exact sequence

$$
0 \rightarrow \operatorname{ker} P(D) \hookrightarrow A(G) \stackrel{P(D)}{\longrightarrow} A(G) \rightarrow 0
$$

is tame exact.

Certain linear topological invariants $(\bar{\Omega})$ and $(\underline{D N})$ have been used by Vogt [34] to characterize the power series spaces of finite type. We need the following "tame" versions which have been used by Langenbruch [17].

1.12. Definition. Let $E$ be a Fréchet space with a fixed fundamental system of seminorms $\left(|\cdot|_{x}\right)_{x<0}$.

(a) $E$ has property $(\underline{D N})_{t}$ if there are $x_{1}<0, C>0$, and $x_{0}<0$ such that for each $x_{0} \leq x<0$ there are $x_{2}<0$ and $C^{\prime}>0$ with

$$
|f|_{x} \leq C^{\prime}|f|_{x_{1}}^{|x| / C}|f|_{x_{2}}^{1-|x| / C}, \quad f \in E .
$$

The property $(\underline{D N})_{t}$ is inherited by linear-tame isomorphisms and by closed subspaces.

(b) $E$ has property $(\bar{\Omega})_{t}$ if for each $x_{1}<0$ there are $C>0$ and $x_{0}<0$ such that for all $x_{0} \leq x<0$ and $x_{2}<0$ there is $C^{\prime}>0$ with

$$
|\mu|_{x}^{*} \leq C^{\prime}\left(|\mu|_{x_{1}}^{*}\right)^{|x| C}\left(|\mu|_{x_{2}}^{*}\right)^{1-|x| C}, \quad \mu \in E^{\prime},
$$

where $\left(|\cdot|_{x}^{*}\right)_{x<0}$ are the dual norms defined in 1.4. A standard calculation (see Wagner [36, Satz 1.9]) shows that this is in fact the property defined in Langenbruch [17, Definition 1.3]. The property $(\bar{\Omega})_{t}$ is inherited by lineartame isomorphisms and by quotients.

Each power series space $\Lambda_{0}(\beta)$ has both properties $(\underline{D N})_{t}$ and $(\bar{\Omega})_{t}$ (see Langenbruch [17, Lemma 1.4]).

The following is the main result of this section (see 1.3 for notation).

1.13. Theorem. For $P \in A^{0} \backslash\{0\}$ and for $G$ as in 1.1, the differential operator $P(D)$ admits a solution operator on $A(G)$ if and only if $\operatorname{ker} P(D)$ has the property $(\bar{\Omega})_{t}$ with respect to the norm system of 1.2 , i.e., if and only if for each 
$x_{1}<0$ there are $C>0$ and $x_{0}<0$ such that for all $x_{0} \leq x<0$ and $x_{2}<0$ there is $C^{\prime}>0$ with

$$
\|q(f)\|_{x} \leq C^{\prime}\|q(f)\|_{x_{1}}^{|x| C}\|q(f)\|_{x_{2}}^{1-|x| C}, \quad f \in A_{H},\|f\|_{x_{1}}<\infty,
$$

$\|q(f)\|_{x}, x<0$, being the quotient "norms" defined in 1.4.

Proof. We have to prove that the short exact sequence

$$
0 \rightarrow \operatorname{ker} P(D) \hookrightarrow A(G) \stackrel{P(D)}{\longrightarrow} A(G) \rightarrow 0
$$

splits if and only if $\operatorname{ker} P(D)$ admits $(\bar{\Omega})_{t}$.

$\Leftarrow:$ By $1.9,1.12$, and the hypothesis, $\operatorname{ker} P(D)$ has properties $(\underline{D N})_{t}$ and $(\bar{\Omega})_{t}$. Checking the proof of Vogt [34, Satz 1.6], which states that $\operatorname{ker} P(D)$ having the properties $(\bar{\Omega})$ and $(\underline{D N})$ is isomorphic to a power series space of finite type, Langenbruch [17, Theorem 1.5], observed that by the particular (linear-tame) choices of $(\bar{\Omega})_{t}$ and $(\underline{D N})_{t}$, this isomorphism is in fact a lineartame one. Thus by 1.9 and 1.11 , the short exact sequence given above is in fact a linear-tame exact sequence of power series spaces of finite type. Hence by Vogt [35, Theorem 5.1], or Poppenberg and Vogt [30, Corollary 6.3], the sequence splits.

$\Rightarrow$ : We modify the proof of Vogt [35, Theorem 5.1]. If the sequence splits, there is a continuous projection $L: A(G) \rightarrow \operatorname{ker} P(D)$ of $A(G)$ onto $\operatorname{ker} P(D)$. By Theorem 1.9, $A(G)$ can be identified with $\Lambda_{0}(\beta)$. Hence by Vogt [35, Lemma 2.1], or Vogt [34, Lemma 5.1], the characteristic of continuity $\sigma_{L}$ : ]$-\infty, 0[\rightarrow]-\infty, 0[$ is convex and nondecreasing, and thus $L$ is linearly tame. This shows that $\operatorname{ker} P(D)$ is linear-tame isomorphic to a quotient of $A(G)$; hence by 1.9 and 1.12 , it has the property $(\bar{\Omega})_{t}$.

By 1.5 and $(1), \operatorname{ker} P(D)$ has property $(\bar{\Omega})_{t}$ if and only if (2) holds.

1.14. Remark. As it follows from [28, Proposition 2.4], $\operatorname{ker} P(D)$ always has the property $(\bar{\Omega})$, i.e. (by 1.9 and Vogt $[34, \operatorname{Satz} 1.6]), \operatorname{ker} P(D)$ is always isomorphic to a power series space of finite type. The problem which we consider in the sequel is to decide whether $\operatorname{ker} P(D)$ has even the more special property $(\bar{\Omega})_{t}$ with respect to the natural norm system $\left(|\cdot|_{x}\right)_{x<0}$ which has been defined in 1.2.

In $\S 3$ we will apply the following inheritance property.

1.15. Lemma. Let $\left(P_{l}\right)_{l \in \mathbb{N}}$ be a sequence in $A^{0} \backslash\{0\}$ which converges to some $P \in A^{0} \backslash\{0\}$ in the topology of $A^{0}$. If $\operatorname{ker} P_{l}(D), l \in \mathbb{N}$, have property $(\bar{\Omega})_{t}$ with uniform constants $C, x_{0}$, and $C^{\prime}$, then also $\operatorname{ker} P(D)$ has property $(\bar{\Omega})_{t}$. Proof. For each $l \in \mathbb{N}$ let $q_{l}: A_{H} \rightarrow A_{H} /\left(P_{l} \cdot A_{H}\right)$ denote the quotient map. Omitting an index $l$, for all $x<0$ and all $f \in A_{H}$ with $\|f\|_{x}<\infty$, we write

$$
\left\|q_{l}(f)\right\|_{x}:=\inf \left\{\left\|f+P_{l} h\right\|_{x} \mid h \in A_{H},\left\|P_{l} h\right\|_{x}<\infty\right\} .
$$

We fix $x<y_{1}<y<0$ and $f \in A_{H}$ with $\|f\|_{x}<\infty$. Let $h \in A_{H}$ with $\|P h\|_{x}<\infty$. Dividing $P h$ by $P$, the Division Theorem 1.6 gives $\|h\|_{y_{1}}<\infty$. Hence by the hypothesis, $\lim _{l \rightarrow \infty}\left\|\left(P_{l}-P\right) h\right\|_{y}=0$. This gives

$$
\left\|q_{l}(f)\right\|_{y} \leq\left\|f+P_{l} h\right\|_{y} \leq\|f+P h\|_{y}+\left\|\left(P_{l}-P\right) h\right\|_{y} ;
$$

hence

$$
\underset{l \rightarrow \infty}{\limsup }\left\|q_{l}(f)\right\|_{y} \leq\|f+P h\|_{y} \leq\|f+P h\|_{x}
$$


and thus

$$
\limsup _{l \rightarrow \infty}\left\|q_{l}(f)\right\|_{y} \leq\|q(f)\|_{x}, \quad x<y<0, f \in A_{H},\|f\|_{x}<\infty .
$$

To prove the converse estimate, we fix $x<y_{2}<y<0$ and $f \in A_{H}$ with $\|f\|_{x}<\infty$. We fix $D>1$. For each $l \in \mathbb{N}$ we choose $h_{l} \in A_{H}$ with $\left\|P_{l} h_{l}\right\|_{x}<$ $\infty$ and $D\left\|q_{l}(f)\right\|_{x} \geq\left\|f+P_{l} h_{l}\right\|_{x}$. Then

$$
\left\|P_{l} h_{l}\right\|_{x} \leq D\left\|q_{l}(f)\right\|_{x}+\|f\|_{x} \leq(D+1)\|f\|_{x}, \quad l \in \mathbb{N} .
$$

Dividing $P_{l} h_{l}$ by $P_{l}$, because of the uniform bounds for $P_{l}$, the Division Theorem 1.6 gives $\sup _{l \in \mathbb{N}}\left\|h_{l}\right\|_{y_{2}}<\infty$. Hence by the hypothesis,

$$
\lim _{l \rightarrow \infty}\left\|\left(P_{l}-P\right) h_{l}\right\|_{y}=0 \text {. }
$$

This gives

$$
\begin{aligned}
D\left\|q_{l}(f)\right\|_{x} & \geq\left\|f+P_{l} h_{l}\right\|_{y} \geq\left\|f+P h_{l}\right\|_{y}-\left\|\left(P_{l}-P\right) h_{l}\right\|_{y} \\
& \geq\|q(f)\|_{y}-\left\|\left(P_{l}-P\right) h_{l}\right\|_{y} ;
\end{aligned}
$$

hence,

$$
D \liminf _{l \rightarrow \infty}\left\|q_{l}(f)\right\|_{x} \geq\|q(f)\|_{y}
$$

and thus, since $D>1$ was arbitrary,

(4) $\quad \liminf _{l \rightarrow \infty}\left\|q_{l}(f)\right\|_{x} \geq\|q(f)\|_{y}, \quad x<y<0, f \in A_{H},\|f\|_{x}<\infty$.

By the hypothesis, for each $x_{1}<0$ there are $C>0$ and $x_{0}<0$ such that for all $x_{0} \leq x<0$ and $x_{2}<0$ there is $C^{\prime}>0$ such that for all $f \in A_{H}$ with $\|f\|_{x_{1}}<\infty$ we have

$$
\left\|q_{l}(f)\right\|_{x} \leq C^{\prime}\left\|q_{l}(f)\right\|_{x_{1}}^{|x| C}\left\|q_{l}(f)\right\|_{x_{2}}^{1-|x| C}, \quad l \in \mathbb{N} .
$$

Applying (3) and (4), we get that for all $\varepsilon>0$ and all $f \in A_{H}$ with $\|f\|_{x_{1}-\varepsilon}<$ $\infty$

This proves (2).

$$
\|q(f)\|_{x+\varepsilon} \leq 2 C^{\prime}\|q(f)\|_{x_{1}-\varepsilon}^{|x| C}\|q(f)\|_{x_{2}-\varepsilon}^{1-|x| C} \text {. }
$$

\section{DifFERENTIAL OPERATORS OF INFINITE ORDER}

In [24] for $N=1$, the evaluation of condition $(\bar{\Omega})_{t}$ for $\operatorname{ker} P(D)$ has been done for arbitrary $P \in A^{0} \backslash\{0\}$. For $N>1$, a corresponding approach gives only sufficient conditions for the existence of a solution operator (Proposition 2.4).

2.1. Definition. (a) For $P \in A^{0}$ we set

$$
V(P):=\left\{z \in \mathbb{C}^{N} \mid P(\bar{z})=0\right\} \text { and } \tilde{V}(P):=\left\{z \in \mathbb{C}^{N} \mid P(z)=0\right\} \text {. }
$$

(b) If $V \subset \mathbb{C}^{N}$ is unbounded, we set

$$
A:=\left\{a \in S \mid \exists z_{j} \in V, j \in \mathbf{N} \text {, with } \lim _{j \rightarrow \infty}\left|z_{j}\right|=\infty \text { and } \lim _{j \rightarrow \infty} \frac{z_{j}}{\left|z_{j}\right|}=a\right\} \text {. }
$$

The cone $V_{\infty}:=\mathbf{R}_{+} A=\{t a \mid t \geq 0, a \in A\}$ is called the tangent cone at infinity of the set $V$. Obviously,

$$
\lim _{z \rightarrow \infty, z \in V} \operatorname{dist}\left(z /|z|, V_{\infty}\right)=\lim _{z \rightarrow \infty, z \in V} \operatorname{dist}(z /|z|, A)=0 ;
$$


hence, $\operatorname{dist}\left(z, V_{\infty}\right)=o(|z|)$ as $z \in V$ tends to infinity.

2.2. Lemma. Let $P$ be a nonconstant polynomial of degree $m$ with principal part $P_{m}$. If $V:=V(P)$, then $V_{\infty}=V\left(P_{m}\right)$.

Proof. Obviously $V_{\infty} \subset V\left(P_{m}\right)$. To prove the other inclusion, fix $a \in V\left(P_{m}\right) \cap$ $S$. Let $n \in \mathbb{N}$. Since $P_{m} \not \equiv 0$, there is $\eta \in S$ such that $\zeta \mapsto P_{m}(a+\zeta \eta)$ does not vanish identically on $C$. Thus we can choose $0<r_{n}<1 / n$ and $\delta>0$ such that $\left|P_{m}(a+\zeta \eta)\right| \geq \delta$ for all $|\zeta|=r_{n}$. Hence there is $\lambda_{n}>n$ with

$$
\left|P\left(\lambda_{n} a+\lambda_{n} \zeta \eta\right)-P_{m}\left(\lambda_{n} a+\lambda_{n} \zeta \eta\right)\right|<\delta \lambda_{n}^{m} \leq\left|P_{m}\left(\lambda_{n} a+\lambda_{n} \zeta \eta\right)\right|
$$

for all $|\zeta|=r_{n}$. Since $P_{m}\left(\lambda_{n} a+\lambda_{n} 0 \eta\right)=0$, by Rouchë's theorem, there is $\left|\zeta_{n}\right|<r_{n}$ such that $P\left(z_{n}\right)=0$ where $z_{n}:=\lambda_{n} a+\lambda_{n} \zeta_{n} \eta$. Since

$$
\left|\frac{z_{n}}{\left|z_{n}\right|}-a\right|=\left|\frac{a\left(1-\left|a+\zeta_{n} \eta\right|\right)+\zeta_{n} \eta}{\left|a+\zeta_{n} \eta\right|}\right| \leq \frac{2\left|\zeta_{n}\right|}{1-\left|\zeta_{n}\right|} \leq 2 /(n-1),
$$

we have $\lim _{n \rightarrow \infty} z_{n} /\left|z_{n}\right|=a$ and thus $a \in V_{\infty}$.

2.3. Notation. By $v_{H}$ we denote the largest plurisubharmonic function on $\mathbb{C}^{N}$ with $v_{H} \leq H$ on $\mathbb{C}^{N}$ such that $v_{H}-\log (1+|z|)$ is bounded from above. This function exists by Siciak [32] and is continuous (see [27, Proposition 1.9]).

2.4. Proposition. Let $P \in A^{0} \backslash\{0\}$, and let $V_{\infty}$ be the tangent cone at infinity of $V:=V(P)$. If $v_{H}=H$ on $V_{\infty} \cap U$ for some neighborhood $U$ of the origin, then $P(D)$ admits a solution operator on $A(G)$.

Proof. Put $\tilde{V}:=\tilde{V}(P)$. We are going to apply [28, Proposition 2.4]. Let $\left.\tilde{r}: \mathbb{C}^{N} \rightarrow\right] 0, \infty[$ be the function from $[28,2.2]$, with $\tilde{r}(z)=o(|z|)$, as $|z|$ tends to infinity. Put $X:=\left\{z \in \mathbb{C}^{N} \mid U(z, \tilde{r}(z)) \cap \tilde{V} \neq \varnothing\right\}$. For $z \in \mathbb{C}^{N}$ we define $\Omega(z):=U(z, \tilde{r}(z))$. If $A^{2}(\Omega(z))$ denotes the Hilbert space of all square integrable analytic functions on $\Omega(z)$, we set $I(\Omega(z)):=(P \cdot A(\Omega(z))) \cap$ $A^{2}(\Omega(z))$ and $E_{\Omega(z)}:=A^{2}(\Omega(z)) / I(\Omega(z))$ where $E_{\Omega(z)}$ is endowed with the quotient norm $|\cdot|_{\Omega(z)}$. By $[28,2.4]$, the dual norms in $A_{H} /\left(P \cdot A_{H}\right)$ may be replaced by

$$
\left\|f+P \cdot A_{H}\right\|_{x}:=\sup _{z \in X}|f| \Omega(z)+\left.I(\Omega(z))\right|_{\Omega(z)} e^{-H_{x}(z)}, \quad x<0, f \in A_{H} .
$$

By the hypothesis and [27, Theorem 1.20], we can choose $C^{\prime}>0$ with

$$
H(\bar{z}) \leq H_{x}(\bar{z})+|x| C^{\prime}|z|, \quad x<0, z \in \tilde{V}_{\infty},
$$

where $\widetilde{V}_{\infty}$ is the tangent cone at infinity of $\tilde{V}$. Since $\operatorname{dist}\left(z, V_{\infty}\right)=o(|z|)$ as $z \in V$ tends to infinity, for each $\varepsilon>0$ there is $C_{\varepsilon}>0$ with

$$
H(\bar{z}) \leq H_{x}(\bar{z})+|x| C^{\prime}(1+\varepsilon)|z|+\varepsilon|z|+C_{\ell}, \quad x<0, z \in X .
$$

Hence for each $x<0$ there is $C_{x}>0$ with

$$
H(\bar{z}) \leq H_{x}(\bar{z})+|x| 2 C^{\prime}|z|+C_{x}, \quad z \in X .
$$

Now let $x_{1}<0$ be given. We choose $C>0$ with $C \min _{a \in S}\left(H(a)-H_{x_{1}}(a)\right) \geq$ $2 C^{\prime}$, and $x_{0}<0$ with $\left|x_{0}\right| C<1$. This gives for all $x_{0} \leq x<0$

$$
-H_{x}(\bar{z}) \leq-H(\bar{z})+|x| C\left(H(\bar{z})-H_{x_{1}}(\bar{z})\right)+C_{x}, \quad z \in X .
$$


Hence for all $x_{0} \leq x<0, x_{2}<0$ and $f \in A_{H}$ with $\|f\|_{x_{1}}<\infty$ we get with $y:=f+P \cdot A_{H}$

$$
\|y\|_{x} \leq e^{C_{x}}\|y\|_{x_{1}}^{|x| C}\|y\|_{0}^{1-|x| C} \leq e^{C_{x}}\|y\|_{x_{1}}^{|x| C}\|y\|_{x_{2}}^{1-|x| C} .
$$

Thus by Theorem 1.13, $P(D)$ admits a solution operator on $A(G)$.

In the case $N=1$, in view of [27, Theorems 1.14 and 1.20], we proved in [24] that the condition of Proposition 2.4 is also necessary for the existence of a solution operator. In $\S 3$ we will show how to weaken the lower bound for $v_{H} \mid V(P)$ to get a criterion for the existence of a solution operator for homogeneous polynomials $P$ on $\mathbb{C}^{N}, N \geq 2$. For particular functions $P$, we will now interpret property $(\bar{\Omega})_{t}$ of $\operatorname{ker} P(D)$ as a Phragmén-Lindelöf property on $\widetilde{V}(P)$ as it has been introduced by Hörmander [11] and as it occurs in Meise, Taylor, and Vogt [21].

2.5. Definition. Let $P \in A^{0} \backslash\{0\}$ and $\tilde{V}:=\tilde{V}(P)$. We consider the space

$$
\begin{gathered}
A_{H}(\tilde{V}):=\left\{f \in A\left(\mathbb{C}^{N}\right) \mid\|f\|_{\widetilde{V}, x}<\infty \text { for some } x<0\right\}, \\
\|f\|_{\widetilde{V}, x}:=\sup _{z \in \widetilde{V}}|f(z)| \exp \left(-H_{x}(\bar{z})\right) .
\end{gathered}
$$

$P$ will be called interpolating if the restriction map $\rho_{\widetilde{V}}: A_{H} \rightarrow A_{H}(\tilde{V})$, $\rho_{\widetilde{V}}(f):=f \mid \widetilde{V}$ is surjective, if there are $C>0$ and $x_{0}<0$ such that for each $x_{0} \leq x<0$ there is $C^{\prime}>0$ with

$$
\inf \left\{\|\tilde{f}\|_{x / C} \mid \tilde{f} \in A_{H}, \rho_{\tilde{V}}(\tilde{f})=f\right\} \leq C^{\prime}\|f\|_{\tilde{V}, x}, \quad f \in A_{H}(\tilde{V}),
$$

and if, moreover, $\operatorname{ker} \rho_{\widetilde{V}}=P \cdot A_{H}$.

2.6. Theorem. Let $G$ and $H$ be as in 1.1. If $P \in A^{0} \backslash\{0\}$ is interpolating according to 2.5 and $\widetilde{V}:=\widetilde{V}(P)$, then the following are equivalent.

(i) $P(D)$ admits a solution operator on $A(G)$.

(ii) $\rho_{\widetilde{V}}$ admits an extension operator, i.e., there is a continuous linear map $E: A_{H}(\tilde{V}) \rightarrow A_{H}$ with $\rho_{\widetilde{V}} \circ E=$ id $_{A_{H}(\widetilde{V})}$.

(iii) For each $x_{1}<0$ there are $C>0$ and $x_{0}<0$ such that for all $x_{0} \leq x<0$ and $x_{2}<0$ there is $C^{\prime}>0$ with

$$
\|f\|_{\widetilde{V}, x} \leq C^{\prime}\|f\|_{\widetilde{v}, x_{1}}^{|x| C}\|f\|_{\widetilde{v}, x_{2}}^{1-|x| C}, \quad f \in A\left(\mathbb{C}^{N}\right),\|f\|_{\widetilde{V}, x_{1}}<\infty .
$$

(iv) For each $x_{1}<0$ there are $C>0$ and $x_{0}<0$ such that for all $x_{0} \leq x<0$ and $x_{2}<0$ there is $C^{\prime}>0$ such that

$$
\log |f(z)| \leq H_{x}(\bar{z})+C K|x|+C^{\prime} \text { for all } z \in \tilde{V},
$$

whenever $f \in A\left(\mathbb{C}^{N}\right)$ and $K>0$ satisfy

$$
\log |f(z)| \leq H_{x_{1}}(\bar{z})+K \text { and } \log |f(z)| \leq H_{x_{2}}(\bar{z}) \text { for all } z \in \widetilde{V} \text {. }
$$

Proof. By Definition 2.5, $\rho_{\widetilde{V}}$ induces a "linear-tame" isomorphism $A_{H}(\tilde{V}) \cong$ $A_{H} /\left(P \cdot A_{H}\right)$. Thus we may identify $A_{H} /\left(P \cdot A_{H}\right)$ and $A_{H}(\tilde{V})$.

(i) $\Leftrightarrow$ (ii): By 1.5 and duality theory for (FS)-spaces.

(i) $\Leftrightarrow$ (iii): By Theorem 1.13. 
(iii) $\Leftrightarrow$ (iv): Because of the "homogeneity" of the inequality, it is enough to know this inequality for all $f \in A\left(\mathbb{C}^{N}\right)$ with $\|f\|_{\tilde{V}, x_{1}}<\infty$ and $\|f\|_{\tilde{V}, x_{2}}=1$. This proves (iii) $\Leftrightarrow\left(\right.$ iv' $^{\prime}$ ), where (iv') is condition (iv) with $K>0$ replaced by $K \in \mathbf{R}$. Of course (iv') implies (iv). On the other hand, (iv) is trivial for $K \leq 0$ : If $\log |f(z)| \leq H_{x_{1}}(\bar{z})+K$ for all $z \in \tilde{V}$, then (assuming $C,\left|x_{1}\right| \geq\left|x_{0}\right|$ ) we get

$$
\log |f(z)| \leq H_{x}(\bar{z})+K|x| / C+K(1-|x| / C) \leq H_{x}(\bar{z})+K|x| / C
$$

for all $z \in \tilde{V}$ and $x_{0} \leq x<0$. Thus (iv) implies (iv').

We give two classes of interpolating functions. The most important one is described in the following proposition which is a consequence of the Ehrenpreis fundamental principle (see Hansen [8]).

2.7. Proposition. Each nonzero polynomial on $\mathbb{C}^{N}$ which is the product of pairwise distinct irreducible factors is interpolating.

2.8. Lemma. Let $\varphi: \mathbf{R}_{+} \rightarrow \mathbf{R}_{+}$be a differentiable nondecreasing and unbounded function such that $\log \varphi$ is convex. Then for each nondecreasing function $f: \mathbb{R}_{+} \rightarrow \mathbb{R}_{+}$with $f(x)=o(\varphi(x))$ as $x \rightarrow \infty$, there is a nondecreasing and convex function $g: \mathbf{R}_{+} \rightarrow \mathbf{R}_{+}$with $f \leq g$ and $g(x)=o(\varphi(x))$ as $x \rightarrow \infty$. Proof. We choose inductively numbers $0<R_{k}<R_{k+1}, k \in \mathbb{N}$, with $f(x) \leq$ $(1 /(k+1)) \varphi(x)$ for all $x \geq R_{k}$ and such that the tangent at $R_{k}$ to the graph of $(1 / k) \varphi$ intersects the graph of $(1 /(k+1)) \varphi$ at a point $R_{k} \leq x_{k} \leq R_{k+1}$ (this is possible because the hypotheses imply $\lim _{x \rightarrow \infty} \phi^{\prime}(x)=\infty$ ). For each $k \in \mathbb{N}$ on $\left[R_{k}, x_{k}\right]$ we connect the graphs of $(1 / k) \varphi$ and $(1 /(k+1)) \varphi$ by this tangent. In this way we get the graph of a nondecreasing function $g: \mathbf{R}_{+} \rightarrow \mathbf{R}_{+}$ with $f(x) \leq g(x)$ for $x \geq R_{1}$ and with $g(x)=o(\varphi(x))$ as $x \rightarrow \infty$. To prove the convexity of $g$, we fix $k \in \mathbb{N}$. Since the tangents are nondecreasing, we have $(1 / k) \varphi\left(R_{k}\right) \leq(1 /(k+1)) \varphi\left(x_{k}\right)$. By the assumption, the function $x \mapsto \varphi^{\prime}(x) / \varphi(x)$ is nondecreasing. Hence

$$
(k+1) / k \leq \varphi\left(x_{k}\right) / \varphi\left(R_{k}\right) \leq \varphi^{\prime}\left(x_{k}\right) / \varphi^{\prime}\left(R_{k}\right) .
$$

This gives $(1 / k) \varphi^{\prime}\left(R_{k}\right) \leq(1 /(k+1)) \varphi^{\prime}\left(x_{k}\right)$ and thus the convexity of $g$. Adding a sufficiently large number to $g$, the assertion is proved.

The following is an extension of a result of Berenstein and Taylor [5]. For similar more-recent results we refer to Papush and Russakovskii [29] and to the literature cited therein.

2.9. Proposition. Let $P \in A^{0}$ be such that for each $\varepsilon>0$ there is $C^{\prime}>0$ with

$$
|\operatorname{grad} P(z)| \geq\left(1 / C^{\prime}\right) \exp (-\varepsilon|z|), \quad z \in \tilde{V}(P) .
$$

Then the function $P$ is interpolating.

Proof. We modify the proof of Berenstein and Taylor [5, Theorem 1]. We put

$$
f(x):=\max \left\{\max _{z \in B\left(e^{x}\right)} \log |P(z)|, \max _{z \in B\left(e^{x}\right), z \in \tilde{V}(P)}-\log |\operatorname{grad} P(z)|, x, 0\right\},
$$


By the hypothesis we have $f(x)=o\left(e^{x}\right)$ as $x \rightarrow \infty$. Applying Lemma 2.8 to $\varphi(x)=e^{x}$, we find a convex and nondecreasing function $g: \mathbf{R} \rightarrow \mathbf{R}_{+}$with $f \leq g$ and $g(x)=o\left(e^{x}\right)$ as $x \rightarrow \infty$. Thus by Hörmander [10, Theorem 1.6.7], $q: \mathbb{C}^{N} \rightarrow \mathbb{R}_{+}, q(z):=g(\log |z|)$, is plurisubharmonic. By the choice of $q$, we have $\log |P| \leq q, \log (1+|z|)=O(q(z))$ as $|z| \rightarrow \infty$, and

$$
|\operatorname{grad} P(z)| \geq \exp (-q(z)), \quad z \in \tilde{V}(P) .
$$

Hence by [5, (14)], there are $A_{1}, B_{1}>0$ such that

$$
|\operatorname{grad} P(z)| \geq\left(1 / A_{1}\right) \exp \left(-B_{1} q\left(B_{1} z\right)\right), \quad z \in S\left(P, B_{1}\right),
$$

where $S\left(P, B_{1}\right)$ denotes the union of those components of the set

$$
\left\{z \in \mathbb{C}^{N}|| P(z) \mid<\exp \left(-B_{1} q\left(B_{1} z\right)\right)\right\}
$$

which meet $\tilde{V}:=\tilde{V}(P)$. Let $x<0$ and $f \in A_{H}(\tilde{V})$ with $\|f\|_{\tilde{V}, x}<\infty$, i.e.,

$$
|f(z)| \leq\|f\|_{\tilde{V}, x} \exp H_{x}(\bar{z}), \quad z \in \tilde{V} .
$$

Checking [5] or just citing Demailly [7, Corollaire 3 and Remarque 7] (where an appropriate holomorphic retract $r: S\left(P, B_{1}\right) \rightarrow V$ is constructed), we get $A_{2}, B_{2}>0$ not depending on $f$ and an extension $F \in A\left(S\left(P, B_{1}\right)\right)$ of $f$ with

$$
\int_{S\left(P, B_{1}\right)}|F(z)|^{2} \exp \left(-2 H_{x}(\bar{z})-B_{2} q\left(B_{2} z\right)\right) d \lambda(z) \leq A_{2}\|f\|_{\widetilde{V}, x}^{2} .
$$

By an obvious modification of the semilocal interpolation theorem of Berenstein and Taylor [4, Theorem 2.2], or by the procedure of the proof of [28, Proposition 2.4], there are $A_{3}, B_{3}>0$ not depending on $f$ and an extension $\tilde{f} \in A\left(\mathbb{C}^{N}\right)$ of $f$ with

$$
\begin{aligned}
& \int_{\mathbf{C}^{N}}|\tilde{f}(z)|^{2} \exp \left(-2 H_{x}(\bar{z})-B_{3} q\left(B_{3} z\right)\right) d \lambda(z) \\
& \quad \leq A_{3} \int_{S\left(P, B_{1}\right)}|F(z)|^{2} \exp \left(-2 H_{x}(\bar{z})-B_{2} q\left(B_{2} z\right)\right) d \lambda(z) .
\end{aligned}
$$

Applying the subharmonicity of $|\tilde{f}|^{2}$, this gives for some $A_{4}, B_{4}>0$ which do not depend on $f$

$$
|\tilde{f}(z)| \leq A_{4}\|f\|_{\tilde{V}, x} \exp \left(H_{x}(\bar{z})+B_{4} q\left(B_{4} z\right)\right), \quad z \in \mathbb{C}^{N} .
$$

Since $q(z)=o(|z|)$ as $|z| \rightarrow \infty$, this shows that 2.4 holds for arbitrary $C>1$. Since grad $P$ does not vanish on $\widetilde{V}$ and by the Division Theorem 1.6, we have $\operatorname{ker} \rho_{\widetilde{V}}=P \cdot A_{H}$ (see Chirka [6, 2.9, Proposition 2]).

In the sequel the following simple lemma is very useful. Its proof only relies on the fact that differential operators commute.

2.10. Lemma. Let $P_{1}, P_{2} \in A^{0} \backslash\{0\}$, and put $P:=P_{1} P_{2}$. Then $P(D)$ admits a solution operator on $A(G)$ if and only if $P_{1}(D)$ and $P_{2}(D)$ do. In particular, if $R: A(G) \rightarrow A(G)$ is a solution operator of $P(D)$, then $R_{1}:=P_{2}(D) \circ R$ is a solution operator for $P_{1}(D)$.

\section{Partial differential operators}

In the present section we study partial differential operators, i.e., differential operators $P(D)$ given by polynomials $P$. 
3.1. Notation. Let $V \subset \mathbb{C}^{N}$ be an analytic variety. Let $V^{0}$ denote the set of regular points of $V$. A function $u: V^{0} \rightarrow[-\infty, \infty[$ is said to be plurisubharmonic on $V$ if $u$ is plurisubharmonic on $V^{0}$ and locally bounded from above on $V$. For $z \in V \backslash V^{0}$ we put $u(z):=\lim \sup _{w \rightarrow z, w \in V 0} u(w)$. Then $u$ is an upper semicontinuous function on $V$ (see Sadullaev [31]).

3.2. Theorem. Let $G$ and $H$ be as in 1.1. Let $P$ be a nonconstant polynomial on $\mathbb{C}^{N}$, and put $V:=V(P)$ and $\widetilde{V}:=\widetilde{V}(P)$. The following assertions are equivalent:

(i) $P(D)$ admits a solution operator on $A(G)$.

(ii) $Q(D)$ admits a solution operator on $A(G)$, where $Q$ is the product of the pairwise distinct irreducible factors of $P$.

(iii) Condition 2.6(iv) holds.

(iv) For each $x_{1}<0$ there are $C>0$ and $x_{0}<0$ such that for all $x_{0} \leq x<0$ and $x_{2}<0$ there is $C^{\prime}>0$ such that

$$
u \leq H_{x}+C K|x|+C^{\prime} \text { on } V(P),
$$

for all plurisubharmonic functions $u$ on $V(P)$ and $K>0$ with

$$
u \leq H_{x_{1}}+K \text { and } u \leq H_{x_{2}} \text { on } V(P) \text {. }
$$

Condition (iv) shows in particular that $P(D)$ admits a solution operator on $A(G)$ if and only if $\bar{P}(D)$ does $\left(\bar{P}(z):=\overline{P(\bar{z})}, z \in \mathbb{C}^{N}\right)$.

Proof. The equivalence of (i) and (ii) holds by Lemma 2.10. Since $\tilde{V}(P)=$ $\widetilde{V}(Q)$, (ii) and (iii) are equivalent by Theorem 2.6 . Let (iv') be the condition (iii), where $\log |f|$ is replaced by any plurisubharmonic function $u$ on $\mathbb{C}^{N}$. Since $u$ is plurisubharmonic if and only if $z \mapsto u(\bar{z})$ is, we have (iv) $\Leftrightarrow\left(\mathrm{iv}^{\prime}\right)$. Since (iv') $\Rightarrow$ (iii) is trivial, it remains to prove (iii) $\Rightarrow\left(\right.$ iv $\left.^{\prime}\right)$. This is done by a general procedure of Meise, Taylor, and Vogt [22]:

We indicate the modifications of the proof of Theorem 2.3 of Meise, Taylor, and Vogt [22], which are necessary to get the announced result. We first note that by 1.1 , (iii) implies a version (iii') of (iii) where $H_{x_{1}}+K$ and $H_{x_{2}}$ are replaced by $H_{x_{1}}+K+\log (2+|z|) /\left|x_{2}\right|$ and $H_{x_{2}}+\log (2+|z|) /\left|x_{2}\right|$, respectively. Now let $\tilde{x}_{1}<x_{1}<0$ be given. We choose $C>0$ and $x_{0}<0$ according to (iii'). Let $x_{0} \leq x<0$ and $\tilde{x}_{2}<x_{2}<0$. Choose $C^{\prime}>0$ according to (iii'). We choose $0<\theta<1$ with $H_{\tilde{x}_{1}} / \theta \leq H_{x_{1}}$ and $H_{\dot{x}_{2}} / \theta \leq H_{x_{2}}$. Let $\tilde{u}$ be plurisubharmonic on $\tilde{V}$, and let $K>0$ satisfy

$$
\tilde{u}(z) \leq H_{\tilde{x}_{1}}(\bar{z})+K \text { and } \tilde{u}(z) \leq H_{\bar{x}_{2}}(\bar{z}) \text { for all } z \in \tilde{V} \text {. }
$$

Put $u:=\tilde{u} / \theta$. In the proof of [22, Theorem 2.3], it has been shown (see [22, p. 303]) that (iii') implies the existence of some constant $B>0$ which does not depend on $\tilde{u}$ or $K$, such that for all $z \in \tilde{V}$

$$
\tilde{u}(z)=\theta u(z) \leq H_{x}(\bar{z})+C K|x|+C^{\prime}+B \log (2+|z|) .
$$

For all $x_{0}<\tilde{x}_{0} \leq x<0$ there are $x_{0} \leq \tilde{x}<x$ with $|\tilde{x}| /|x| \leq 2$ and a constant $C^{\prime \prime}>0$ which does not depend on $\tilde{u}$ or $K$ such that for all $z \in \tilde{V}$

$$
\begin{aligned}
\tilde{u}(z) & \leq H_{\tilde{x}}(\bar{z})+C K|\tilde{x}|+C^{\prime}+B \log (2+|z|) \\
& \leq H_{x}(\bar{z})+\frac{|\tilde{x}|}{|x|} C K|x|+C^{\prime \prime} \leq H_{x}(\bar{z})+2 C K|x|+C^{\prime \prime} .
\end{aligned}
$$


Since the criterion becomes nicer if we confine ourselves to homogeneous polynomials, we are interested in the following two results.

3.3. Proposition. Let $P$ be a nonconstant polynomial on $\mathbb{C}^{N}$ of degree $m$ with principal part $P_{m}$. If $P(D)$ admits a solution operator on $A(G)$, then also $P_{m}(D)$ does.

Proof. Let $Q$ be the product of the distinct irreducible factors of $P$. Let $n$ be its degree. The polynomials $\tilde{Q}_{l}(z)=l^{-n} Q(l z), z \in \mathbb{C}^{N}, l \in \mathbb{N}$, converge to the principal part $Q_{n}$ of $Q$ in the topology of $A^{0}$. By Proposition 2.7, the polynomials $\tilde{Q}_{l}, l \in \mathbb{N}$, and $Q$ are interpolating. By the hypothesis and Lemma 2.10, $Q$ satisfies 2.6 (iii). If we apply condition 2.6(iii) to the functions $z \mapsto f(z / l)^{l}$ on $\widetilde{V}(Q)$, this shows that for each $x_{1}<0$ there are $C>0$ and $x_{0}<0$ such that for all $x_{0} \leq x<0$ and $x_{2}<0$ there is $C^{\prime}>0$ with

$$
\|f\|_{\widetilde{v}_{l}, x} \leq C^{1 / l}\|f\|_{\widetilde{v}_{l}, x_{1}}^{|x| C}\|f\|_{\widetilde{v}_{l}, x_{2}}^{1-|x| C}, \quad f \in A\left(\mathbb{C}^{N}\right),\|f\|_{\widetilde{v}_{l}, x_{1}}<\infty, l \in \mathbb{N},
$$

where $\tilde{V}_{l}:=\tilde{V}\left(\tilde{Q}_{l}\right)$. By Definition 2.5 and Theorem 1.13, $\operatorname{ker} \tilde{Q}_{l}(D), l \in \mathbb{N}$, have property $(\bar{\Omega})_{t}$ with uniform constants. By Lemma 1.15 , also $\operatorname{ker} Q_{n}(D)$ has property $(\bar{\Omega})_{t}$. By Theorem $1.13, Q_{n}(D)$ admits a solution operator on $A(G)$, and thus by Lemma 2.10 , so does $P_{m}(D)$.

\subsection{Proposition. For $N=2$, the converse of Proposition 3.3 holds.}

Proof. Compare Meise, Taylor and Vogt [21, Theorem 4.11]. The author thanks R. W. Braun for a hint to prove the result. Let $V:=V(P)$ and $V_{m}:=V\left(P_{m}\right)$. Note that $V_{m}$ is a finite union of complex lines passing through the origin and which only intersect there. By the Puiseux expansion (see, e.g., Hörmander [12, A.1]), there are $\widetilde{R} \geq R>0$ and a multivalued analytic map $\varphi: V_{m} \backslash B(R) \rightarrow V$, continuous on $V_{m} \mid U(R)$, with $|\varphi(z)-z|=o(|z|)$ as $|z| \rightarrow \infty$ and such that $V \backslash B(\widetilde{R}) \subset \varphi\left(V_{m} \backslash B(R)\right.$ ) (compare [21, Theorem 4.11]). The essential property of the multivalued analytic map $\varphi$ is that for each $z \in V_{m} \backslash B(R)$ there are a neighborhood $U$ of $z$ in $V_{m}$ and holomorphic functions $f_{1}, \ldots, f_{n}$ on $U$ such that $\varphi(w)=\left\{f_{1}(w), \ldots, f_{n}(w)\right\}$ for all $w \in U$. Let $\tilde{x}_{1}<x_{1}<0$ be given. Choose $x_{0}$ and $C>0$ according to 3.2(ii) (for $V_{m}$ ). Let $x_{0} \leq$ $x<0$ and $\tilde{x}_{2}<x_{2}<0$, choose $C^{\prime}>0$ according to 3.2 (ii), and put $M:=$ $\max _{w \in V_{m} \cap \theta B(R)} H_{\bar{x}_{2}}(w)$. Fix $K>0$ and a plurisubharmonic function $u$ on $V$ satisfying $u \leq H_{\dot{x}_{1}}+K$ and $u \leq H_{\tilde{x}_{2}}$ on $V$. We set $\tilde{u}(z):=\max \{u(\varphi(z)), M\}$ for $z \in V_{m} \backslash B(R)$ and $\tilde{u}:=M$ on $V_{m} \cap B(R)$. Here $u(\varphi(z))$ is defined as the maximum over all finitely many values of $\varphi(z)$. Then $\tilde{u}$ is plurisubharmonic on $V_{m}$. Since $|\varphi(z)-z|=o(|z|)$ as $|z| \rightarrow \infty$ and by 1.1 , there is $M^{\prime} \geq M$ not depending on $u$ or $K$ such that

$$
\tilde{u}-M^{\prime} \leq H_{x_{1}}+K \text { and } \tilde{u}-M^{\prime} \leq H_{x_{2}} \text { on } V_{m} .
$$

By 3.2(ii), we get

$$
u(\varphi(z)) \leq \tilde{u}(z) \leq H_{x}(z)+C K|x|+C^{\prime}+M^{\prime}, \quad z \in V_{m} \backslash B(R) .
$$

Hence for all $x_{0}<\tilde{x}_{0} \leq x<0$ there are $x_{0} \leq \tilde{x}<x$ with $|\tilde{x}| /|x| \leq 2$ and some $C^{\prime \prime}>0$ which does not depend on $u$ or $K$ such that

$$
u(w) \leq H_{x}(w)+\frac{|\tilde{x}|}{|x|} C K|x|+C^{\prime \prime}, \quad w \in V \backslash B(\tilde{R}),
$$


and thus

$$
u \leq H_{x}+2 C K|x|+C^{\prime \prime}+\sup _{w \in B(\widetilde{R})} H_{\tilde{x}_{2}}(w) \text { on } V .
$$

In the sequel we will investigate homogeneous polynomials only.

3.5. Notation. Let $V \subset \mathbb{C}^{N}$ be an algebraic variety which is homogeneous, i.e., $\mathbb{C} \cdot V=V$. For $C>0$, we denote by $C \mathscr{L}_{V}$ the class of all plurisubharmonic functions $u$ on $V$ such that $u(z)-C \log (1+|z|), z \in V$, is bounded from above. Extending Notation 2.3, we define

$$
v_{H}(z ; C, V):=\sup _{u} u(z), \quad z \in V,
$$

where the supremum is taken over all $u \in C \mathscr{L}_{V}$ with $u \leq H$ on $V$. By Siciak [32] (see [27, Proposition 1.9]), this extremal function is again contained in $C \mathscr{L}_{V}$ and does not exceed $H$. Since $\log |z|=o(H(z))$ as $|z| \rightarrow \infty$, for each $C>0$ there is $d>0$ with $v_{H}(z ; C, V) \geq C \log |z|-d$ for all $z \in V$.

We consider the compact set $P_{H, V} \subset V$ starshaped with respect to the origin and the positively homogeneous function $C_{H, V}: V \rightarrow[0, \infty]$ (i.e., $C_{H, V}(\lambda z)=\lambda C_{H, V}(z)$ for all $z \in V, \lambda \geq 0$ ) which is defined by

$$
P_{H, V}:=\left\{z \in V \mid v_{H}(z ; 1, V)=H(z)\right\}=\left\{\lambda a \mid a \in S \cap V, 0 \leq \lambda \leq 1 / C_{H, V}(a)\right\} .
$$

The following definition makes sense even if $H$ is the support function of a convex set which does not contain the origin as an interior point (for example, for a convex domain of $\mathbf{R}^{N}$ ). For $\varepsilon, r>0$, we define the following plurisubharmonic function on $V \cap U(r)$

$$
u_{H}(z ; r, \varepsilon, V):=\sup _{u} u(z), \quad z \in V \cap U(r),
$$

where the supremum is taken over all plurisubharmonic functions $u$ on $V \cap U(r)$ with $u \leq H$ on $V \cap U(r)$ and $u^{*}(z):=\limsup _{\zeta \rightarrow z} u(\zeta) \leq H(z)-\varepsilon$ for all $z \in V \cap \partial U(r)$.

A particular role is played by the cone

$$
\begin{aligned}
\Gamma_{H, V} & :=\left\{t a \mid t \geq 0, a \in P_{H, V}\right\} \\
& =\left\{z \in \mathbb{C}^{N} \mid v_{H}(z ; C, V)=H(z) \text { for some } C>0\right\} .
\end{aligned}
$$

If $V=\mathbb{C}^{n}$ or $C=1$, respectively, we will omit the index $V$ and $C$, respectively,

The behavior of the functions $u_{H}$ and $v_{H}$ near the origin is essential for our purposes.

3.6. Lemma. Let $V$ be as in 3.5. For all $\varepsilon>0$ and $r>0$ there is some $C>0$ such that

$$
u_{H}(\cdot ; r, \varepsilon, V) \leq v_{H}(\cdot ; C, V) \text { on } V \cap U(r) \text {. }
$$

Conversely, for all $C, E>0$ there is $r>0$ such that

$$
v_{H}(\cdot ; C, V) \leq u_{H}(\cdot ; r, E, V) \text { on } V \cap U(r) \text {. }
$$

Proof. Let $\varepsilon>0$ and $r>0$. The proof of [27, Lemma 2.1] also applies to plurisubharmonic functions on $V$ (instead of $\mathbb{C}^{N}$ ). Thus there is $C>0$ with

$$
u_{H}(\cdot ; r, \varepsilon, V) \leq v_{H}(\cdot ; C, V) \text { on } U(r) \cap V \text {. }
$$

On the other hand, let $C, E>0$. Since $v_{H}(\cdot ; C, V) \in C \mathscr{L}_{V}$ and since $|z|=O(H(z))$ for $|z| \rightarrow \infty$, there is some $r>0$ with $v_{H}(z ; C, V) \leq H(z)-E$ for $|z| \geq r$. 
3.7. Theorem. Let $G, H$ be as in 1.1. If $P$ is a nonzero homogeneous polynomial on $\mathbb{C}^{N}$, then the following assertions are equivalent.

(i) $P(D)$ admits a solution operator on $A(G)$.

(ii) For each $x_{1}<0$ there are $C>0$ and $x_{0}<0$ such that

$$
\log |f| \leq \inf _{x_{0} \leq x<0}\left(H_{x}+C K|x|\right) \text { on } V(P),
$$

whenever $f \in A\left(\mathbb{C}^{N}\right)$ and $K>0$ satisfy

$$
\log |f| \leq H_{x_{1}}+K \text { and } \log |f| \leq H \text { on } V(P) \text {. }
$$

(iii) For each $x_{1}<0$ there are $C>0$ and $x_{0}<0$ such that

$$
u \leq \inf _{x_{0} \leq x<0}\left(H_{x}+C|x|\right) \text { on } V(P) \text {, }
$$

for each plurisubharmonic function $u$ on $V(P)$ with

$$
u \leq H_{x_{1}}+1 \text { and } u \leq H \text { on } V(P) \text {. }
$$

(iv) For all $\varepsilon>0, r>0$ and $E>0$ there is $R>0$ such that

$u_{H}(\cdot ; r, \varepsilon, V(P)) \leq u_{H}\left(\cdot ; R, E, \mathbb{C}^{N}\right)=: u_{H}(\cdot ; R, E) \quad$ on $V(P) \cap U(r)$.

(v) There are $\delta>0$ and a neighborhood $U$ of the origin such that

$$
v_{H}(\cdot ; \delta, V(P)) \leq v_{H} \text { on } V(P) \cap U .
$$

(vii) There is $\delta>0$ such that

$$
v_{H}(\cdot ; \delta, V(P)) \leq v_{H} \text { on } V(P) .
$$

Proof. By Theorem 3.2, $P$ satisfies 3.2(iii) if and only if $\bar{P}$ satisfies 3.2(iii). We may thus switch from $\widetilde{V}(P)$ to $V(P)$ in 3.2(iii). Let (iii') be the assertion of 3.2(iv) however, with $H_{x_{2}}$ replaced by $H$ and $C^{\prime}$ replaced by 0 . If we consider the plurisubharmonic functions $z \mapsto u(K z) / K, z \in V(P)$, we obtain that (iii) and (iii') are equivalent. By Theorem 3.2, we have (iii') $\Rightarrow$ (ii) $\Rightarrow$ 3.2(iii) $\Rightarrow$ (i) $\Rightarrow 3.2$ (iv). To show that 3.2(iv) implies (iii'), for each $x_{1}>0$ let $C>0$ and $x_{0}$ be chosen according to 3.2(iv). Then for all $x_{0} \leq x<0$ and $x_{2}<0$ there is $C^{\prime} \geq 0$ such that the conclusion of 3.2(iv) holds. For each plurisubharmonic function $u$ on $V(P)$ we apply the conclusion of 3.2(iv) to the functions $\lambda u(\cdot / \lambda), \lambda>0$, and obtain that we may choose $C^{\prime}=0$. Let now $u$ be plurisubharmonic on $V(P)$ with $u \leq H_{x_{1}}+1$ and $u \leq H$ on $V(P)$. Then for each $0<\eta<1$ there is $x_{2}<0$ with $\eta H \leq H_{x_{2}}$. Thus $\eta u \leq H_{x_{1}}+1$ and $\eta u \leq H_{x_{2}}$. By 3.2(iv), we obtain $\eta u \leq \inf _{x_{0} \leq x<0}\left(H_{x}+C|x|\right)$ on $V(P)$. In the limit for $\eta \rightarrow 1$ we get our claim. By Theorem 3.2, we have thus proved (i) $\Leftrightarrow$ (ii) $\Leftrightarrow$ (iii) .

(iii') $\Rightarrow$ (iv): Let $\varepsilon, r>0$ and $E>0$. We choose $x_{1}<0$ with $H-\varepsilon \leq H_{x_{1}}$ on $\partial U(r)$. Put $K:=\max _{z \in B(r)} H_{x_{1}}(z)$. Fix a plurisubharmonic function $\tilde{u}$ on $V(P) \cap U(r)$ with $\tilde{u} \leq H$ on $V(P) \cap U(r)$ and $\tilde{u}^{*} \leq H-\varepsilon$ on $V(P) \cap \partial U(r)$. We put $u:=\max \left\{\tilde{u}, H_{x_{1}}\right\}$ on $V(P) \cap U(r)$ and $u:=H_{x_{1}}$ on $V(P) \backslash U(r)$. Then $u$ is plurisubharmonic on $V(P)$ with $u \leq H_{x_{1}}+K$ and $u \leq H$ on $V(P)$. By (iii'), we get

$$
\tilde{u} \leq u \leq \inf _{x_{0} \leq x<0}\left(H_{x}+C K|x|\right)=\inf _{x_{0} \leq x<0}\left(H_{x}-C K x\right) \text { on } V(P) \cap U(r),
$$


and thus by the definition of $u_{H}$

$$
u_{H}(\cdot ; r, \varepsilon, V(P)) \leq \inf _{x_{0} \leq x<0}\left(H_{x}-C K x\right) \text { on } V(P) \cap U(r) \text {. }
$$

By the minimum principle of Kiselman [15, 4.1] (see [27, Proposition 1.3]), the right-hand side is plurisubharmonic on $\mathbb{C}^{N}$ and does not exceed $H$. If we choose $R \geq r$ with $H_{x_{0}}+C K\left|x_{0}\right| \leq H-E$ on $\partial U(R)$, we obtain

$$
u_{H}(\cdot ; r, \varepsilon, V(P)) \leq u_{H}(\cdot ; R, E) \text { on } V(P) \cap U(r) \text {. }
$$

(iv) $\Rightarrow$ (v): By (iv) and by Lemma 3.6, there are $R \geq r>0$ and $C \geq 1$ such that for all $z \in V(P) \cap U(r)$

$$
v_{H}(z ; 1, V(P)) \leq u_{H}(z ; r, 1, V(P)) \leq u_{H}(z ; R, 1) \leq v_{H}(z ; C) .
$$

This gives $v_{H}(\cdot ; 1 / C, V(P)) \leq v_{H}(\cdot ; 1)=v_{H}$ on $V(P) \cap U(r / C)$.

(v) $\Rightarrow\left(\right.$ vi): Since $v_{H}-\log (1+|z|)$ is bounded from below, we may choose by [27, Proposition 1.9], some $0<\delta^{\prime}<\min \{1, \delta\}$ such that $v_{H}\left(\cdot ; \delta^{\prime}, V(P)\right) \leq$ $v_{H}$ on $V(P)$ outside $U$.

(vi) $\Rightarrow$ (iv): Let $\varepsilon, E, r>0$. By (vi) and Lemma 3.6, there are $C, C^{\prime}>0$ and $R>0$ such that for all $z \in V(P) \cap U(r)$

$$
u_{H}(z ; r, \varepsilon, V(P)) \leq v_{H}(z ; C, V(P)) \leq v_{H}\left(z ; C^{\prime}\right) \leq u_{H}(z ; R, E) .
$$

(iv) $\Rightarrow$ (iii): Let $x_{1}<0$ be given. We choose $x_{1}<x_{0}<0$ and $r>0$ with $H_{x_{1}}+1 \leq H-1$ and $H_{x_{1}}+1 \leq H_{x_{0}}$ on $\mathbb{C}^{N} \backslash U(r)$. We fix a plurisubharmonic function $u$ on $V(P)$ with

$$
u \leq H_{x_{1}}+1 \text { and } u \leq H \text { on } V(P) \text {. }
$$

By (iv) and Lemma 3.6, there are $C>0$ and $R \geq r$ such that for all $z \in$ $V(P) \cap U(r)$

$$
u(z) \leq u_{H}(z ; r, 1, V(P)) \leq u_{H}(z ; R, 1) \leq v_{H}(z ; C) .
$$

By [27, Theorem 1.20], there is $C^{\prime} \geq C$ with $v_{H}(\cdot ; C) \leq \inf _{x<0}\left(H_{x}+C^{\prime}|x|\right)$ on $\mathbf{C}^{N}$. Thus

$$
u \leq \inf _{x<0}\left(H_{x}+C^{\prime}|x|\right) \leq \inf _{x_{0} \leq x<0}\left(H_{x}+C^{\prime}|x|\right) \quad \text { on } V(P) \cap U(r) .
$$

On the other hand,

$$
u \leq H_{x_{1}}+1 \leq H_{x_{0}} \leq \inf _{x_{0} \leq x<0}\left(H_{x}+C^{\prime}|x|\right) \text { on } V(P) \backslash U(r) .
$$

Hence $u \leq \inf _{x_{0} \leq x<0}\left(H_{x}+C^{\prime}|x|\right)$ on $V(P)$.

We get the following necessary condition for the existence of a solution operator.

3.8. Theorem. Let $P$ be a nonconstant polynomial on $\mathbb{C}^{N}$ with principal part $P_{m}$. Then (i) $\Rightarrow$ (ii) $\Rightarrow$ (iii) $\Rightarrow$ (iv) (see 3.5 for the notation):

(i) $P(D)$ admits a solution operator on $A(G)$.

(ii) There is $\delta>0$ with $v_{H}\left(\cdot ; \delta, V\left(P_{m}\right)\right) \leq v_{H}$ on $V\left(P_{m}\right)$.

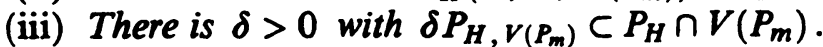

(iv) $\Gamma_{H, V\left(P_{m}\right)} \subset \Gamma_{H} \cap V\left(P_{m}\right)$ (even $\left.{ }^{\omega}="\right)$. 
Proof. (i) $\Rightarrow$ (ii): Proposition 3.3 and Theorem 3.7. The remaining implications are obvious.

The following resembles a result of Grothendieck which states that no elliptic partial differential operator admits a solution operator on the space $C^{\infty}(\Omega)$ for $\boldsymbol{\Omega} \subset \mathbf{R}^{N}$ open.

3.9. Corollary. Let $P$ be a nonconstant polynomial on $\mathbb{C}^{N}$ with principal part $P_{m}$. If $V\left(P_{m}\right) \cap \Gamma_{H}=\{0\}$ (see 3.5), then the partial differential operator $P(D)$ admits no solution operator on $A(G)$.

Proof. Assume that $P(D)$ admits a solution operator on $A(G)$. Then $\Gamma_{H, V\left(P_{m}\right)} \subset \Gamma_{H} \cap V\left(P_{m}\right)=\{0\}$, by Theorem 3.8. On the other hand, since $\log |z|=o(H(z))$ as $|z| \rightarrow \infty$, there is $d:=\inf _{z \in V\left(P_{m}\right)}(H(z)-\log |z|) \in \mathbb{R}$. Since $H$ is continuous and $V\left(P_{m}\right)$ is closed, there is $a \in V\left(P_{m}\right) \backslash\{0\}$ with $d=H(a)-\log |a|$. Hence $v_{H}\left(a ; 1, V\left(P_{m}\right)\right)=H(a)$ and $a \in \Gamma_{H, V\left(P_{m}\right)}$.

The following result roughly says that for a polyhedron $G$ the equivalent conditions of 3.7 hold if and only if $v_{H} \mid V(P)$ is a maximal plurisubharmonic function on $V(P) \backslash \Gamma_{H}$. It justifies calling 3.2(iii) and 3.2(iv) a Phragmén-Lindelöf condition.

Let $W$ be an analytic variety. We recall that a plurisubharmonic function $v$ on $W$ is called "maximal" if $u \leq v$ holds for each open set $U \subset \subset W$ and each plurisubharmonic function $u$ on $U$ with $\lim \sup _{\zeta \rightarrow z} u(\zeta) \leq v(z)$ for all $z \in \partial U$ (see Klimek [16]).

3.10. Proposition. Let $C_{H}$ be bounded on $\Gamma_{H} \cap S$. By [27, Theorem 2.11], this holds for instance if $G$ is a polyhedron. Then $\Gamma_{H}$ is a closed cone and for each nonzero homogeneous polynomial $P$ on $\mathbb{C}^{N}$, the following are equivalent.

(i) There is $\delta>0$ with $v_{H}(\cdot ; \delta, V(P)) \leq v_{H}$ on $V(P)$.

(ii) There are $\delta>0$ and a plurisubharmonic function $v$ on $V(P)$ with $v_{H}(\cdot ; \delta) \leq v \leq v_{H}$ on $V(P)$, which is maximal on $V(P) \backslash \Gamma_{H}$.

Proof. Let $V:=V(P)$.

(i) $\Rightarrow$ (ii) : Put $v:=v_{H}(\cdot ; \delta, V)$. First let $P$ be linear. By standard arguments (see Bedford and Taylor [3, Corollary 9.2]) and by Bedford and Taylor [2, §9], we obtain $\left(d d^{c} v\right)^{N-1}=0$ on $V \backslash \Gamma_{H, V}$ (see [27, Proposition 1.9]). By Theorem 3.8, we have $\Gamma_{H, V}=\Gamma_{H} \cap V$. Hence $\left(d d^{c} v\right)^{N-1}=0$ on $V \backslash \Gamma_{H}$. By Bedford's and Taylor's domination theorem [2,3], $v$ is maximal on $V \backslash \Gamma_{H}$. The same procedure works if $V$ is a smooth variety. In the general case of a variety $V$ having singularities, we obtain by this procedure that $\left(d d^{c} v\right)^{N-1}=0$ holds on $V^{0} \backslash \Gamma_{H}$, where $V^{0}$ is the smooth variety of regular points. By the extended domination theorem of Bedford [1] and Zeriahi [38, 1.9], $v$ is maximal on $V \backslash \Gamma_{H}$.

(ii) $\Rightarrow$ (i): We will apply Theorem $3.7(\mathrm{v})$. By $3.5, v_{H}(\cdot ; 1, V)$ grows at most like $\log |z|$ and $v_{H}(\cdot ; 2)$ grows at least like $2 \log |z|$. Hence we may choose $R>0$ such that $u:=v_{h}(\cdot ; 1, V) \leq v_{H}(\cdot ; 2)$ on $V \backslash U(R)$. Since $C_{H}$ is bounded on $\Gamma_{H} \cap S$, there is $C_{1} \geq 2$ such that $v_{H}\left(\cdot ; C_{1}\right)=H$ on $\Gamma_{H} \cap B(R)$. The function $\tilde{v}:=v\left(\cdot / C_{1}\right) C_{1}$ is plurisubharmonic on $V$ maximal on $V \backslash \Gamma_{H}$ and satisfies

$$
\begin{aligned}
v_{H}\left(z ; C_{1}\right) & =C_{1} v_{H}\left(z / C_{1} ; 1\right) \leq \tilde{v}(z) \leq C_{1} v_{H}\left(z / C_{1} ; C\right) \\
& =v_{H}\left(z ; C_{1} C\right), \quad z \in V .
\end{aligned}
$$


By the choice of $R$ and since $C_{1} \geq 2$, we get

$$
u \leq v_{H}(\cdot ; 2) \leq v_{H}\left(\cdot ; C_{1}\right) \text { on } V \cap \partial B(R)
$$

and

$$
u \leq H=v_{H}\left(\cdot ; C_{1}\right) \text { on } V \cap B(R) \cap \Gamma_{H} .
$$

Thus by the continuity of $v_{H}\left(\cdot ; C_{2}\right)$, we obtain for all $z \in \partial\left((V \cap U(R)) \backslash \Gamma_{H}\right)$ (with respect to $V$ )

$$
\begin{aligned}
\liminf _{\zeta \rightarrow z}(\tilde{v}(\zeta)-u(\zeta)) & \geq \liminf _{\zeta \rightarrow z}\left(v_{H}\left(\zeta ; C_{1}\right)-u(\zeta)\right) \\
& =v_{H}\left(z ; C_{1}\right)-u^{*}(z)=v_{H}\left(z ; C_{1}\right)-u(z) \geq 0 .
\end{aligned}
$$

By the hypothesis (see Klimek [16, Proposition 3.1]), we conclude

$$
v_{H}(\cdot ; 1, V)=u \leq \tilde{v} \leq v_{H}\left(\cdot ; C_{1} C\right) \text { on } V \cap U(R) \text {. }
$$

We are going to evaluate condition 3.7(vi). In general this is a nontrivial problem even in the case of linear functionals $P$ (which are enough to consider many examples of partial differential operators). We thus restrict the discussion to very simple domains $G$, which are the Cartesian product of plain polygons. To prove Theorem 3.14 we need some preparations.

3.11. Lemma. Assume that $G=\prod_{l=1}^{n} G_{j}$, where $G_{l} \subset \mathbb{C}^{N_{l}}$ has the support function $H_{l}, l=1, \ldots, n, N=\sum_{l=1}^{n} N_{l}$. Then there are $C, C^{\prime}>0$ with $v_{H} \leq v \leq v_{H}(\cdot ; C)$ for $v(z):=\sum_{l=1}^{n} v_{H_{l}}\left(z_{l} ; C^{\prime}\right), z \in \mathbb{C}^{N}=\prod_{l=1}^{n} \mathbb{C}^{N_{l}}$. Moreover, $\Gamma_{H}=\prod_{l=1}^{n} \Gamma_{H_{l}}$.

Proof. The last assertion holds by [27, Lemma 2.6]. By 3.5, there are $C_{l}>0$, $l=1, \ldots, n$, with $v_{H_{l}}\left(z_{l} ; 2\right) \geq 2 \log \left|z_{l}\right|-C_{l}, z_{l} \in \mathbb{C}$. We put $E:=2 \log \sqrt{N}+$ $\max _{l=1}^{n} C_{l}$. Since $v_{H}$ grows at most like $\log |z|$ for $|z| \rightarrow \infty$, there is $R>0$ with

$$
v_{H}(z) \leq 2 \log |z|-E, \quad z \in \mathbb{C}^{N},|z| \geq R .
$$

Hence we obtain for $|z| \geq R$

$$
\begin{aligned}
v_{H}(z) & \leq 2 \max _{l=1}^{n} \log \left|z_{l}\right|+2 \log \sqrt{N}-E \\
& \leq \max _{l=1}^{n} v_{H_{l}}\left(z_{l} ; 2\right) \leq \sum_{l=1}^{n} v_{H_{l}}\left(z_{l} ; 2\right) .
\end{aligned}
$$

By the proof of [27, Lemma 2.6], we have

$$
\sum_{l=1}^{n} v_{H_{l}}\left(z_{l}\right) \leq v_{H}(z ; n), \quad z \in \mathbb{C}^{N},
$$

and that there is $D \geq 2$ such that

$$
v_{H}(z) \leq \sum_{l=1}^{n}\left(v_{H_{l}}\left(z_{l} ; D\right)+\sum_{k \neq l} H_{k}\left(z_{k}\right)\right) / n=\sum_{l=1}^{n} u_{l}\left(z_{l}\right), \quad z \in B(R),
$$

where for each $l=1, \ldots, n$

$$
u_{l}\left(z_{l}\right):=\left(v_{H_{l}}\left(z_{l} ; D\right)+(n-1) H_{l}\left(z_{l}\right)\right) / n, \quad z_{l} \in \mathbb{C}^{N_{l}} .
$$


We choose $R^{\prime} \geq R$ such that $v_{H_{l}}\left(z_{l} ; D\right) \leq H_{l}\left(z_{l}\right)-1$ for all $\left|z_{l}\right| \geq R^{\prime}, l=$ $1, \ldots, n$. Applying [27, Lemma 2.1], to $u_{l}$ for each $l=1, \ldots, n$, we get $C^{\prime} \geq 2$ with

$$
u_{l}\left(z_{l}\right) \leq v_{H_{l}}\left(z_{l} ; C^{\prime}\right), \quad\left|z_{l}\right| \leq R^{\prime},
$$

and hence

$$
v_{H}(z) \leq \sum_{l=1}^{n} v_{H_{l}}\left(z_{l} ; C^{\prime}\right), \quad|z| \leq R^{\prime} .
$$

For $|z| \geq R^{\prime}$ this estimate holds by the choice of $R$. If we put $C:=D C^{\prime}$, we get the assertion.

3.12. Lemma. Assume that $G$ has the form $G=\prod_{j=1}^{N} G_{j}$ where $G_{j} \subset \mathbb{C}$ is a polygon for each $j=1, \ldots, N$. Let $P$ be a nonzero linear functional on $\mathbb{C}^{N}$ and $V:=V(P)$. The following assertions (and hence those of Theorem 3.8) are equivalent.

(i) There is $\delta>0$ with $v_{H}(\cdot ; \delta, V) \leq v_{H}$ on $V$.

(ii) $\Gamma_{H, V}=\Gamma_{H} \cap V$.

(iii) $H \mid V$ is maximal on $V \backslash \Gamma_{H}$ (see Proposition 3.10).

Proof. Let $H_{j}$ be the support function of $G_{j}, j=1, \ldots, N$. (i) $\Rightarrow$ (ii) holds by Theorem 3.8.

(ii) $\Rightarrow$ (iii): Since $V$ is a subspace of $\mathbb{C}^{N}$ of dimension $N-1$ and $G$ is a polyhedron, by [27, Theorem 2.11], the support of $\left(d d^{c} H \mid V\right)^{N-1}$ is contained in $\Gamma_{H, V}$. By the domination theorem of Bedford and Taylor [2], $H \mid V$ is maximal on $V \backslash \Gamma_{H, V}=V \backslash \Gamma_{H}$.

(iii) $\Rightarrow$ (i): We will apply Proposition 3.10. Let $v$ be chosen according to Lemma 3.11. We are going to prove that $v \mid V$ is maximal on $V \backslash \Gamma_{H}$. Fix $a \in V \backslash \Gamma_{H}$. Since $V$ is a subspace of $\mathbb{C}^{N}$ and $G$ is a polyhedron and since $H \mid V$ is maximal on $V \backslash \Gamma_{H}$, by [25, Proposition 9], there are $\eta \in V \cap S$ and a neighborhood $U$ of zero in $\mathbb{C}$ such that the map $\zeta \mapsto H(a+\zeta \eta)=\sum_{j=1}^{N} H_{j}\left(a_{j}+\right.$ $\left.\zeta \eta_{j}\right), \zeta \in U$, is affine. For simplicity we assume that $\eta_{1}, \ldots, \eta_{l} \neq 0$ and $\eta_{l+1}=\cdots=\eta_{N}=0 \quad(1 \leq l \leq N)$. Then for each $j=1, \ldots, l$ also the function $\zeta \mapsto H_{j}\left(a_{j}+\zeta \eta_{j}\right)$ is harmonic (and hence affine) on $U$. It follows from the definition of $v_{H_{j}}$ by a standard argument that hence the function $v_{H_{j}}\left(\cdot ; C^{\prime}\right)$ is harmonic on a neighborhood of $a_{j}$ for each $j=1, \ldots, l$. This shows that $u: z \mapsto v(z)-\sum_{j=1}^{l} v_{H_{j}}\left(z_{j}, C^{\prime}\right), z \in \mathbb{C}^{N}$, is plurisubharmonic and does not depend on $\left(z_{1}, \ldots, z_{l}\right)$ on a neighborhood of $a$. Since we may consider $\eta \in \mathbb{C}^{l} \times\{0\}$ as one vector of a basis of $V$, we obtain that $u \mid V$ depends only on $N-2$ coordinates in a neighborhood $U^{\prime}$ of $a$ in $V$. Hence $\left(d d^{c} u \mid V\right)^{N-1}=0$ there. Since $z \mapsto \sum_{j=1}^{l} v_{H_{j}}\left(z_{j}, C^{\prime}\right)$ is pluriharmonic on $U^{\prime}$, we obtain that $\left(d d^{c} v \mid V\right)^{N-1}=0$ on $U^{\prime}$. By the domination theorem of Bedford and Taylor [2], $v \mid V$ is maximal on $V \backslash \Gamma_{H}$; and by Lemma 3.11, the function $v \mid V$ satisfies the hypothesis of Proposition 3.10.

3.13. Lemma. Let $G$ be as in Lemma 3.12, let $P(z)=\sum_{j=1}^{N} n_{j} z_{j}, z \in \mathbb{C}^{N}$, for some $n \in \mathbb{C}^{N} \backslash\{0\}$; and let $H_{i}$ be the support function of $G_{i}, i=1,2$.

(a) If $N=2$, then $H \mid V(P)$ is maximal (i.e., harmonic) on $V(P) \backslash \Gamma_{H}$ if and only if $n_{1}=0$ or $n_{2}=0$ or $n_{2} \Gamma_{H_{1}}=-n_{1} \Gamma_{H_{2}}$. 
(b) If $N \geq 3$, then $H \mid V(P)$ is maximal on $V(P) \backslash \Gamma_{H}$ if and only if there is $1 \leq j_{0} \leq N$ with $P(z)=n_{j_{0}} z_{j_{0}}, z \in \mathbb{C}^{N}$.

Proof. By Lemma 3.11, we have $\Gamma_{H}=\prod_{j=1}^{N} \Gamma_{H_{j}}$. Set $V:=V(P)$.

(a) $\Rightarrow$ : If $n_{1} \neq 0 \neq n_{2}$, we put $\alpha:=-n_{2} / n_{1}$. Assume that there is $w_{2} \in$ $P_{H_{2}} \backslash\{0\} \subset \Gamma_{H_{2}}$ such that $\bar{\alpha} w_{2} \notin \Gamma_{H_{1}}$. Then $\left(\bar{\alpha} w_{2}, w_{2}\right) \in V \backslash \Gamma_{H}$. By the hypothesis, the convex function

$$
z_{2} \mapsto H\left(\bar{\alpha} z_{2}, z_{2}\right)=H_{1}\left(\bar{\alpha} z_{2}\right)+H_{2}\left(z_{2}\right), \quad z_{2} \in \mathbb{C},
$$

is harmonic in a neighborhood of $w_{2}$. Hence also $\mathrm{H}_{2}$ is harmonic and hence affine in a neighborhood of $w_{2}$. By the homogeneity of $H_{2}$ and [27, Lemma 2.5], this is a contradiction to $w_{2} \in P_{H_{2}} \backslash\{0\}$. Thus we have proved $\bar{\alpha} \Gamma_{H_{2}}=$ $\bar{\alpha} \mathbb{R}_{+} P_{H_{2}} \subset \Gamma_{H_{1}}$ and $-n_{1} \Gamma_{H_{2}} \subset n_{2} \Gamma_{H_{1}}$. The other inclusion can be proved in the same way.

$\Leftarrow:$ If $-n_{1} \Gamma_{H_{2}}=n_{2} \Gamma_{H_{1}}$ and in particular $n_{1} \neq 0 \neq n_{2}$, we set $\alpha:=-n_{2} / n_{1}$. Then $V=\left\{\left(\bar{\alpha} z_{2}, z_{2}\right) \mid z_{2} \in \mathbb{C}\right\}$. Fix $w=\left(\bar{\alpha} w_{2}, w_{2}\right) \in V \backslash\left(\Gamma_{H_{1}} \times \Gamma_{H_{2}}\right)$. If $w_{2} \notin \Gamma_{H_{2}}$, then by the hypothesis, also $\bar{\alpha} w_{2} \notin \Gamma_{H_{1}}$ (and vice versa). Hence $H_{2}$ and $z_{2} \mapsto H_{1}\left(\bar{\alpha} z_{2}\right)$ are harmonic in a neighborhood of $w_{2}$. This shows that $H \mid V$ is harmonic in a neighborhood of $w$. If $n_{1}=0$ or $n_{2}=0$, we argue as in part (b).

(b) $\Rightarrow$ : Choose $1 \leq j_{0} \leq N$ with $n_{j_{0}} \neq 0$. To simplify the notation, we may assume that $j_{0}=N$. We put $\alpha_{j}:=-n_{j} / n_{N}, j=1, \ldots, N-1$. Then

$$
V=\left\{w=\left(w^{\prime}, w_{N}\right) \in \mathbb{C}^{N} \mid w_{N}=w_{N}\left(w^{\prime}\right):=\sum_{j=1}^{N-1} \bar{\alpha}_{j} w_{j}\right\} .
$$

Assume that there is $j_{1} \neq N$, say, $j_{1}=N-1$, with $n_{j_{1}} \neq 0$. Fix $w^{\prime}=$ $\left(w_{1}, \ldots, w_{N-1}\right) \in\left(\mathbb{C} \mid \Gamma_{H_{1}}\right) \times \mathbb{C}^{N-2}$. Since $w=\left(w^{\prime}, w_{N}\left(w^{\prime}\right)\right) \in V \backslash \Gamma_{H}, H \mid V$ is maximal in a neighborhood of $w$. Since $V$ is a subspace of $\mathbb{C}^{N}$ and $G$ is a polyhedron, by [25, Proposition 9], $H$ is affine on a complex line in $V$ through the point $w$. Hence there is $\eta \in \mathbb{C}^{N-1} \backslash\{0\}$ such that

$$
\zeta \mapsto \sum_{j=1}^{N-1} H_{j}\left(w_{j}+\eta_{j} \zeta\right)+H_{N}\left(w_{N}\left(w^{\prime}+\eta \zeta\right)\right), \quad \zeta \in \mathbb{C},
$$

is affine in a neighborhood of 0 . Since $H$ is convex and $\eta \neq 0$, also the function $\zeta \mapsto H_{N}\left(w_{N}\left(w^{\prime}+\eta \zeta\right)\right)$ is affine in a neighborhood of 0 , i.e., $H_{N}$ is affine in a neighborhood of $w_{N}\left(w^{\prime}\right)$. This implies that $w_{N}\left(w^{\prime}\right) \in \mathbb{C} \backslash \Gamma_{H_{N}}$. This gives

$$
\mathbb{C}=\left(\bar{\alpha}_{1} \mathbb{C} \backslash \Gamma_{H_{1}}\right)+\sum_{j=2}^{N-1} \bar{\alpha}_{j} \mathbb{C} \subset \mathbb{C} \backslash \Gamma_{H_{N}} \neq \mathbb{C},
$$

which is a contradiction. Thus $P(z)=n_{N} z_{N}=n_{j_{0}} z_{j_{0}}$ for all $z \in \mathbb{C}^{N}$.

$\Leftarrow$ : If $P(z)=n_{j_{0}} z_{j_{0}}$ for all $z \in \mathbb{C}^{N}$, say, $j_{0}=N$, then $V=\mathbb{C}^{N-1} \times\{0\}$. By [26, Lemma 3.4], $H \mid V$ is maximal on

$$
\left(\mathbb{C}^{N-1} \backslash \Gamma_{\prod_{j=1}^{N-1} G_{j}}\right) \times\{0\}=\left(\mathbb{C}^{N-1} \backslash \prod_{j=1}^{N-1} \Gamma_{H_{j}}\right) \times\{0\}=V \backslash \Gamma_{H}
$$


We are now ready to evaluate condition 3.7 (vi) in the most simple nontrivial situation. In this special case all conditions of Theorem 3.8 are equivalent. We do not expect that this holds in general. For the following result compare Meise, Taylor, and Vogt [21, Theorem 4.11].

3.14. Theorem. Let $G=G_{1} \times G_{2} \subset \mathbb{C}^{2}$ where $G_{i}$ is a bounded open convex polygon in $\mathbb{C}$ which contains the origin and with support function $H_{i}, i=1,2$. Let $P$ be a nonconstant polynomial on $\mathbb{C}^{2}$ of degree $m$ with principal part $P_{m}$. Put $V_{m}:=V\left(P_{m}\right)$. Let the numbers $\alpha_{1}, \ldots, \alpha_{n} \in \mathbb{C} \backslash\{0\}$ be determined by the representation

$$
P_{m}(z)=c z_{1}^{l_{1}} z_{2}^{l_{2}} \prod_{j=1}^{n}\left(z_{1}-\alpha_{j} z_{2}\right), \quad z \in \mathbb{C}^{2},
$$

for appropriate $c \in \mathbb{C} \backslash\{0\}$ and $l_{1}, l_{2} \in \mathbb{N}_{0}$. Then the following are equivalent.

(i) The partial differential operator $P(D)$ admits a solution operator on $A(G)$.

(ii) There is $C>0$ with $C_{H} \leq C C_{H, V_{m}}$ on $V_{m}$.

(iii) $\Gamma_{H, V_{m}}=\Gamma_{H} \cap V_{m}$.

(iv) $H \mid V_{m}$ is harmonic on $V_{m} \backslash \Gamma_{H}$.

(v) $\alpha_{j} \Gamma_{H_{1}}=\Gamma_{H_{2}}$ for all $j=1, \ldots, n$.

In this special case $\Gamma_{H_{i}}$, which is the support of the Laplacian of $H_{i}$, equals the cone which is generated by the outer normals to the faces of $G_{i}, i=1,2$.

Proof. Since in any case (ii) is equivalent with 3.8(iii), the implications (i) $\Rightarrow$ (ii) $\Rightarrow$ (iii) hold by Theorem 3.8. Since $V_{m}$ is the union of the complex lines $\overline{\alpha_{j}} z_{2}=z_{1}, j=1, \ldots, n$, plus perhaps $\mathbb{C} \times\{0\}$ or $\{0\} \times \mathbb{C}$, the only singular point of $V_{m}$ is the origin. Hence the conditions (iii), (iv), and (v) hold if and only if they hold for each linear factor of $P_{m}$. Thus by Lemmas 3.12 and 3.13, we have (iii) $\Leftrightarrow$ (iv) $\Leftrightarrow$ (v). Again by Lemma 3.12 and Lemma 2.10, (iii) implies that $P_{m}(D)$ has a solution operator on $A(G)$. By Proposition 3.4, this implies (i).

3.15. Examples. Let $G=G_{1} \times G_{2} \subset \mathbb{C}^{2}$ be a product of open bounded convex polygons in $\mathbb{C}$. Let $\Gamma_{i}$ be the cone generated by the outer normals to the faces of $G_{i}, i=1,2$.

(i) Let $P(D)=\partial / \partial z_{1}+i \partial / \partial z_{2}$ be the Cauchy-Riemann operator. $P(D)$ admits a solution operator on $A(G)$ if and only if $-i \Gamma_{1}=\Gamma_{2}$.

(ii) Let $P(D)=\partial^{2} / \partial z_{1}^{2}+\partial^{2} / \partial z_{2}^{2}$ be the Laplace operator. Since $P(z)=$ $\left(z_{1}-i z_{2}\right)\left(i z_{1}+i z_{2}\right), z \in \mathbb{C}^{2}$, it admits a solution operator on $A(G)$ if and only if $i \Gamma_{1}=\Gamma_{2}$ and $\Gamma_{1}=-\Gamma_{1}$.

(iii) Let $P(D)=\partial^{2} / \partial z^{2}-\partial^{2} / \partial z_{2}^{2}$ be the d'Alembert operator. Since $P(z)=$ $\left(z_{1}-z_{2}\right)\left(z_{1}+z_{2}\right), z \in \mathbb{C}^{2}$, it admits a solution operator on $A(G)$ if and only if $\Gamma_{1}=\Gamma_{2}$ and $\Gamma_{1}=-\Gamma_{1}$.

(iv) The operator $P(D)=\partial^{2} / \partial z_{1}^{2}-\partial / \partial z_{2}$ of the heat equation admits a solution operator on $A(G)$.

3.16. Examples. Let $G=\prod_{j=1}^{3} G_{j} \subset \mathbb{C}^{3}$ be a product of open bounded convex polygons in $\mathbb{C}$.

(i) Let $P(D)=\partial^{2} / \partial z_{1}^{2}+\partial^{2} / \partial z_{2}^{2}-\partial / \partial z_{3}$ be the operator for the heat equation. By Proposition 3.3, Lemma 2.10, Theorem 3.8, and Lemmas 3.12 and 3.13, $P(D)$ admits no solution operator on $A(G)$. 
(ii) By the same reasoning, the Cauchy-Riemann operator, the Laplace operator, and the d'Alembert operator for the first two variables admit no solution operator on $A(G)$.

\section{REFERENCES}

1. E. Bedford, The operator $\left(d d^{c}\right)^{n}$ on complex spaces, Seminaire Lelong-Skoda, Lecture Notes in Math., vol. 919, Springer, 1982, pp. 294-323.

2. E. Bedford and B. A. Taylor, The Dirichlet problem for a complex Monge-Ampere equation, Invent. Math. 37 (1976), 1-44.

3.

4. C. A. Berenstein and B. A. Taylor, Interpolation problems in $\mathbf{C}^{N}$ with application to harmonic analysis, J. Analyse Math. 38 (1980), 188-254.

5. __, On the geometry of interpolating varieties, Seminaire Lelong-Skoda, Lecture Notes in Math., vol. 919, Springer, 1982, pp. 1-25.

6. E. M. Chirka, Complex analytic sets, Kluwer, 1989.

7. J.-P. Demailly, Scindage holomorphe d'un morphisme de fibres vectoriels semi-positifs avec estimations $L^{2}$, Seminaire Lelong-Skoda, Lecture Notes in Math., vol. 919, Springer, 1982, pp. 77-107.

8. S. Hansen, On the "fundamental principle" of L. Ehrenpreis, Partial Differential Equations, Banach Center Publ., vol. 10, PWN, 1983, pp. 185-201.

9. L. Hörmander, On the range of convolution operators, Ann. of Math. (2) 76 (1962), 148-170.

10. __ An introduction to complex analysis in several variables, Princeton Univ. Press, 1967.

11. On the existence of real analytic solutions of partial differential equations with constant coefficients, Invent. Math. 21 (1973), 151-182.

12. __ The analysis of linear partial differential operators. II, Springer, 1983.

13. G. M. Khenkin and V. S. Mityagin, Linear problems of complex analysis, Russian Math. Surveys 26 (1971), 99-164.

14. C. O. Kiselman, Existence and approximation theorems for solutions of complex analogues of boundary problems, Ark. Mat. 6 (1965), 193-207.

15. $\ldots$ The partial Legendre transform for plurisubharmonic functions, Invent. Math. 49 (1978), 137-148.

16. M. Klimek, Pluripotential theory, Oxford Univ. Press, 1991.

17. M. Langenbruch, Solution operators for partial differential equations in weighted Gevrey spaces, Michigan Math. J. 37 (1990), 3-24.

18. B. Malgrange, Existence et approximation des solutions des équations aux dérivées partielles et des équations de convolution, Ann. Inst. Fourier (Grenoble) 6 (1955-56), 271-355.

19. A. Martineau, Équations différentielles d'ordre infini, Bull. Soc. Math. France 95 (1967), 109-154.

20. R. Meise and B. A. Taylor, Each non-zero convolution operator on the entire functions admits a continuous linear right inverse, Math. Z. 197 (1988), 139-152.

21. R. Meise, B. A. Taylor, and D. Vogt, Characterization of the linear partial differential operators with constant coefficients that admit a continuous linear right inverse, Ann. Inst. Fourier (Grenoble) 40 (1990), 619-655.

22. _Lquivalence of analytic and plurisubharmonic Phragmén-Lindelöf principles on algebraic varieties, Proc. Sympos. Pure Math., vol. 52, Part 3, Amer. Math. Soc., Providence, RI, 1991, pp. 287-308.

23. R. Meise and D. Vogt, Einführung in die Funktionanalysis, Vieweg, 1992.

24. S. Momm, Convex univalent functions and continuous linear right inverses, J. Funct. Anal. 103 (1992), 85-103.

25. _ـ A Phragmén-Lindelöf theorem for plurisubharmonic functions on cones in $\mathbf{C}^{N}$, Indiana Univ. Math. J. 41 (1992), 861-867. 
26. __ A division problem in the space of entire functions of exponential type, Ark. Mat. 32 (to appear).

27. $\ldots$ Boundary behavior of extremal plurisubharmonic functions, Acta Math. 172 (1994), $51-75$.

28. $\ldots$ A critical growth rate of the pluricomplex Green function, Duke Math. J. 72 (1993), 487-502.

29. D. E. Papush and A. M. Russakowskii, Interpolation on plane sets in $\mathbf{C}^{2}$, Ann. Fac. Sci. Toulouse 1 (1992), 337-362.

30. M. Poppenberg and D. Vogt, A tame splitting theorem for exact sequences of Frechet spaces, Math. Z. (to appear).

31. A. Sadullaev, An extimate for polynomials on analytic sets, Math. USSR-Izv. 20 (1983), 493-502.

32. J. Siciak, Extremal plurisubharmonic functions on $C^{N}$, Ann. Polon. Math. 39 (1981), 175-211.

33. F. Tritves, Linear partial differential equations with constant coefficients, Gordon and Breach, 1966.

34. D. Vogt, Eine Charakterisierung der Potenzreihenräume von endlichem Typ und ihre Folgerungen, Manuscripta Math. 37 (1982), 269-301.

35. __, Operators between Frechet spaces, preprint.

36. M. J. Wagner, Quotientenräume von stabilen Potenzreihenräumen endlichen Typs, Manuscripta Math. 31 (1980), 97-109.

37. V. P. Zaharjuta, Extremal phurisubharmonic functions, Hilbert scales and isomorphisms of spaces of analytic functions, I, II, Theor. Funcii, Funktsional Anal. i Prilozhen. vyps. 19, 21 (1974), 133-157, 65-83. (Russian)

38. A. Zeriahi, Fonction de Green pluricomplexe de pole à l'infini sur un espace de Stein parabolique et applications, Math. Scand. 69 (1991), 89-126.

Mathematisches Institut, Hennrich-Henne-Universttät DÜsseldorf, UniversttätsSTRAsSE 1, 40225 DÜsseldorf, Germany

E-mail address: mommemx. cs.uni-duesseldorf.de 